\title{
Relative normal modes for nonlinear Hamiltonian systems *
}

\author{
Juan-Pablo Ortega ${ }^{\dagger}$
}

\begin{abstract}
An estimate on the number of distinct relative periodic orbits around a stable relative equilibrium in a Hamiltonian system with continuous symmetry is given. This result constitutes a generalization to the Hamiltonian symmetric framework of a classical result by Weinstein and Moser on the existence of periodic orbits in the energy levels surrounding a stable equilibrium. The estimate obtained is very precise in the sense that it provides a lower bound for the number of relative periodic orbits at each prescribed energy and momentum values neighboring the stable relative equilibrium in question and with any prefixed (spatiotemporal) isotropy subgroup. Moreover, it is easily computable in particular examples. It is interesting to see how in our result the existence of non trivial relative periodic orbits requires (generic) conditions on the higher order terms of the Taylor expansion of the Hamiltonian function, in contrast with the purely quadratic requirements of the Weinstein-Moser Theorem, which emphasizes the highly non linear character of the relatively periodic dynamical objects.
\end{abstract}

\section{Introduction}

The search for periodic orbits around non hyperbolic equilibria of a Hamiltonian system has traditionally been one of the main topics in classical mechanics. The best known results in this direction are due to Liapounov [38] and Horn [26], who solved the non resonant case. The general case was solved only in 1973 by A. Weinstein who proved the following theorem [67]:

Theorem 1.1 (Weinstein) Let $(M, \omega, h)$ be a Hamiltonian system and let $m \in M$ be an equilibrium of the associated Hamiltonian vector field $X_{h}$ such that $h(m)=0$ and the quadratic form $\mathbf{d}^{2} h(m)$ is definite. Then, for each sufficiently small positive $\epsilon$, there are at least $\frac{1}{2} \operatorname{dim} M$ geometrically distinct periodic orbits of energy $\epsilon$.

Further extensions of this result due to J. Moser [51] justify why this theorem is usually referred to as the Weinstein-Moser Theorem. Bartsch 7 has studied periodic orbits on the zero level set of the Hamiltonian in Moser's generalized version of the theorem. In the last two references, the definiteness of the second variation of the Hamiltonian is not required in the total space but in a smaller subspace called resonance space that will be defined later on in the paper.

In this paper we will be interested in Hamiltonian systems endowed with a continuous symmetry. More specifically, Hamiltonian systems of the form $\left(M, \omega, G, \mathbf{J}: M \rightarrow \mathfrak{g}^{*}, h: M \rightarrow \mathbb{R}\right)$, where $G$ is a Lie group, with Lie algebra $\mathfrak{g}$, acting properly and canonically on the smooth symplectic manifold $(M, \omega)$, that encodes the symmetries of the system. We will assume that the $G$-action admits an equivariant momentum map $\mathbf{J}: M \rightarrow \mathfrak{g}^{*}$, where $\mathfrak{g}^{*}$ denotes the dual space of $\mathfrak{g}$, and that the Hamiltonian function $h$ is $G$-invariant (check for instance with [1] for an introduction to these notions). The generalization of Liapounov's Theorem to the resonant case carried out in the Weinstein-Moser Theorem is of great

\footnotetext{
*This paper is dedicated to the memory of Joaquín Ortega Lacasa.

${ }^{\dagger}$ Institut Nonlinéaire de Nice, UMR 129, CNRS-UNSA, 1361, route des Lucioles, 06560 Valbonne, France. JuanPablo.Ortega@inln.cnrs.fr.
} 
relevance in this setup since the invariance properties associated to symmetries induce resonances in many occasions.

The Weinstein-Moser Theorem was adapted to this category by Montaldi et al 49] and later by Bartsch 6], who obtained sharper estimates. Even though these authors worked in the symmetric framework, their papers still dealt with the search of periodic orbits near elliptic equilibria. However, in the presence of a continuous symmetry, the critical elements that generalize equilibria and periodic orbits to this category are the so-called relative equilibria $(R E)$ and relative periodic orbits (RPOs). Recall that a relative equilibrium of the $G$-invariant Hamiltonian $h$ is a point $m \in M$ such that the integral curve $m(t)$ of the Hamiltonian vector field $X_{h}$ starting at $m$ equals $\exp (t \xi) \cdot m$ for some $\xi \in \mathfrak{g}$, where $\exp : \mathfrak{g} \rightarrow G$ is the exponential map; any such $\xi$ is called a velocity of the relative equilibrium. Note that if $m$ has a non-trivial isotropy subgroup, $\xi$ is not uniquely determined. The point $m \in M$ is said to be a relative periodic orbit of the $G$-invariant Hamiltonian $h$ if there is a $\tau>0$ and an element $g \in G$ such that $F_{t+\tau}(m)=g \cdot F_{t}(m)$ for any $t \in \mathbb{R}$, where $F_{t}$ is the flow of the Hamiltonian vector field $X_{h}$. The constant $\tau>0$ is called the relative period of $m$ and the group element $g \in G$ its phase shift. For historical reasons, we will occasionally refer to the RPOs that we will find in this paper as relative normal modes (we already did so in the title) given that these solutions generalize to the symmetric context the normal modes or periodic orbits around equilibria provided by the Liapounov Center Theorem and by the Weinstein-Moser estimates.

From the point of view of applications a theorem linking stable relative equilibria to the existence of RPOs presents certain relevance since stable relative equilibria are known to appear profusely in most common symmetric Hamiltonian systems: pendula and oscillators subjected to various interactions 42, symmetric rigid bodies (free, in the presence gravity [37, or immersed in fluids [33, 34]), molecules [48], point vortices in various phase spaces 39,32 , etc.

The search for relative equilibria around stable and unstable relative equilibria has been the object of [56. The simplest and most straightforward generalization of the Weinstein-Moser Theorem to the symmetric context is obtained by using symplectic reduction 43 . If the point $m \in M$ is such that $\mathbf{J}(m)=\mu$ is a regular value of the momentum map $\mathbf{J}$ and the coadjoint isotropy subgroup $G_{\mu}$ of $\mu \in \mathfrak{g}^{*}$ acts freely and properly on the level set $\mathbf{J}^{-1}(\mu)$, then the quotient manifold $\mathbf{J}^{-1}(\mu) / G_{\mu}$ is a symplectic manifold and the dynamics of any $G$-invariant Hamiltonian on $M$ drops naturally to Hamiltonian dynamics on the reduced manifold $\mathbf{J}^{-1}(\mu) / G_{\mu}$. Moreover, REs and RPOs in $M$ coincide with equilibria and periodic orbits in the reduced space, respectively. Therefore, if $m$ is a RE such that the Hessian of the reduced Hamiltonian at the reduced equilibrium satisfies the hypothesis of the Weinstein-Moser Theorem, then there are at least $\frac{1}{2} \operatorname{dim}\left(\mathbf{J}^{-1}(\mu) / G_{\mu}\right)$ geometrically distinct periodic orbits on each energy level in this reduced space, that lift to as many geometrically distinct RPOs in $M$ with momentum $\mu$. We emphasize that when in the symmetric context we talk about geometrically distinct objects we mean that one cannot be obtained from the other by using the relevant group action in the problem.

One limitation of this method is that it only allows us to prove the existence of RPOs with the same momentum as the stable relative equilibrium whose existence we use as hypothesis. Additionally, if the regularity assumption on the point $m$ is dropped in the previous paragraph, the reduced space $\mathbf{J}^{-1}(\mu) / G_{\mu}$ is not a manifold anymore but a Poisson variety in the sense of [4, 53], whose symplectic leaves are the singular reduced spaces introduced by Sjamaar, Lerman, and Bates in 62, 86. See also [52, 55]. In principle, the procedure described in the previous paragraph can still be carried out taking, instead of the entire reduced space, the smooth symplectic stratum that contains the reduced equilibrium. The main inconvenience of this approach is the loss of information that the restriction to the stratum implies. For instance, the stratum could reduce to a point, in which case the result would be empty of content. However, even when the stratum that contains the relative equilibrium is not trivial the use of the Weinstein-Moser Theorem in it does not give us any information on the neighboring strata which, as we will see, contain non trivial relative periodic solutions. A first step in overcoming these difficulties has been taken in 36] where a symplectic version of the Slice Theorem due to Marle, Guillemin, and Sternberg is used to establish a relation between the RPOs around a given stable relative equilibrium and the RPOs around the corresponding symmetric equilibrium in the slice, always when certain hypotheses on the coadjoint isotropy of the momentum value of the relative equilibrium are satisfied. This procedure allows the application of the Weinstein-Moser Theorem on 
the slice to produce RPOs of the original system. Nevertheless, this treatment is not optimal and when the relative equilibrium is just an equilibrium, these theorems do not provide any information, as far as RPOs is concerned. In our work we will take an approach inspired by the so called Smale Program 63 . that consists of analyzing the orbit spaces resulting from quotienting the level sets of the conserved quantities in the system by the relevant group action. This strategy will provide results free from some of the restrictions in 36] and containing easy to compute estimates on the number of RPOs around a given stable relative equilibrium, classified by their energy, momentum value, and (spatiotemporal) isotropy subgroups. The main results are contained in the following three theorems:

- Theorem 3.1 this result gives two different lower bounds for the number of RPOs with prescribed energy, momentum, and isotropy group in a symmetric Hamiltonian system, neighboring a stable equilibrium with total isotropy. The symmetry group is assumed to be compact. The estimates provided are based on two different critical point theory tools: Lusternik-Schnirelman category and Morse theory. The former provides an easy to compute dimensional estimate while the latter is expressed in terms on an Euler characteristic with respect to equivariant cohomology that, even though is more difficult to compute it is, in principle, sharper. This result improves the study carried out in [36] since the main results regarding RPOs in those papers are empty of content when dealing with an equilibrium with total isotropy.

- Theorem 3.13 it provides estimates on the number of RPOs similar to those in Theorem 3.1 but this time, the predicted solutions have prescribed isotropy subgroup not only with respect to the symmetry group $G$ of the system (referred to as the group of spatial symmetries) but with respect to the group $G \times S^{1}$ (group of spatiotemporal symmetries), where the circle symmetry comes from putting the system in normal form. This symmetry, that in principle is not a feature of the given system, is reflected in the spatio-temporal symmetry properties of the periods of the solutions predicted by the theorem. Due to the techniques used in the proofs and the conclusions obtained Theorem [3.13 is NOT a generalization of Theorem [3.1] in the proof of Theorem [3.1 intervenes a transversality argument that guarantees that all the solutions obtained are genuine RPOs and not just relative equilibria (that could be considered as trivial RPOs). This conclusion cannot be drawn from Theorem 3.13 given that the subgroups of $G \times S^{1}$ intertwine the $G$ and $S^{1}$-actions via the temporal character (this terminology will be introduced later on) preventing us from making the distinction between RPOs and relative equilibria (see Remark 3.17).

- Theorem 4.1 it generalizes Theorem 3.13 providing, under certain hypotheses, estimates on the number of RPOs around a stable relative equilibrium.

The paper is organized as follows:

- In Section 2 we introduce some preliminary material with the purpose of fixing the notation and of future reference. The expert can skip this section.

- In Section 3 we present the main results that provide an estimate on the number of RPOs surrounding a given stable symmetric equilibrium at each prescribed energy and momentum values neighboring the equilibrium, and with any prefixed spatial and spatiotemporal isotropy subgroup.

- In Section 4 we use the main results in the previous section and the so called reconstruction equations in order to generalize them to an estimate on the number of RPOs around a genuine stable RE.

\section{Preliminaries}

Throughout the paper we will work in the category of symmetric Hamiltonian spaces whose objects are Hamiltonian systems with symmetry $\left(M, \omega, G, \mathbf{J}: M \rightarrow \mathfrak{g}^{*}, h: M \rightarrow \mathbb{R}\right)$. Here $(M, \omega)$ is a symplectic manifold on which the Lie group $G$, with Lie algebra $\mathfrak{g}$, acts properly, canonically, and, moreover, in a globally Hamiltonian fashion, that is, it admits an equivariant momentum map $\mathbf{J}: M \rightarrow \mathfrak{g}^{*}$, where $\mathfrak{g}^{*}$ is the dual space of $\mathfrak{g}$. The Hamiltonian function $h$ is always assumed to be $G$-invariant. 


\subsection{Proper actions, fixed point sets, slices, and normalizers.}

The proofs of the facts stated below can be found in [9, 28, 57, 16]. The isotropy subgroups associated to a proper action are always compact. Let $K$ be a closed subgroup of $G$. The connected components of the sets

$$
\begin{aligned}
M^{K} & =\left\{z \in M \mid K \subseteq G_{z}\right\} \\
M_{K} & =\left\{z \in M \mid K=G_{z}\right\}
\end{aligned}
$$

are submanifolds of $M . M_{K}$ is an open submanifold of $M^{K} . M^{K}$ is usually referred to as the set of $K$-fixed points in $M$ and $M_{K}$ as the submanifold of isotropy type $K$. If $M$ is a symplectic manifold, then (the connected components of) $M_{K}$ and $M^{K}$ are symplectic submanifolds of $M$. Proper actions are important since they guarantee the existence of slices and tubular models: let $m \in M$ and $G_{m}$ be the isotropy subgroup of the element $m$. The Slice Theorem guarantees the existence of a $G$-equivariant isomorphism $\varphi: G \times \times_{G_{m}} B \longrightarrow U$, where $U$ is a $G$-invariant open neighborhood of the orbit $G \cdot m$ such that $\varphi[e, 0]=m$, and where $B$ is an open $G_{m}$-invariant neighborhood of 0 in the vector space $T_{m} M / T_{m}(G \cdot m)$, on which $G_{m}$ acts linearly by $h \cdot\left(v+T_{m}(G \cdot m)\right):=T_{m} \Phi_{h} \cdot v+T_{m}(G \cdot m)$. The set $S$ defined as $S:=\varphi[e, B]$, is a (smooth) slice at $m$ for the $G$-action on $M$.

Slices have very interesting properties. A feature that will be of particular interest interest to us is the possibility of using the slice to locally coordinatize the $G$-space $M$ around the orbit $G \cdot m$ by means of a local cross-section of $G / G_{m}$. More specifically, a local cross-section $\sigma$ of the homogeneous space $G / G_{m}$ is a differentiable map $\sigma: \mathcal{Z} \rightarrow G$, where $\mathcal{Z}$ is an open neighborhood of $G_{m}$ in $G / G_{m}$ such that $\sigma\left(G_{m}\right)=$ e and $\sigma(z) \in z$, for $z \in \mathcal{Z}$. The Slice Theorem for proper actions of Palais [57] propositions 2.1.2 and 2.1.4] guarantees that the map $F: \mathcal{Z} \times S \rightarrow M$ defined by $F(z, s):=\sigma(z) \cdot s$, is a diffeomorphism onto an open subset of $M$ that contains $m$. In Section 4 we will briefly review a symplectic version of this result.

We now suppose that the symplectic manifold $M$ in question is a symplectic vector space $(V, \omega)$ that constitutes a symplectic representation space of $G$. In this case the $K$-fixed point space $V^{K}$ is a symplectic vector subspace of $V$, for any subgroup $K \subset G$. Recall that any symplectic representation is globally Hamiltonian with an equivariant momentum map $\mathbf{J}: V \rightarrow \mathfrak{g}^{*}$ associated given by

$$
\langle\mathbf{J}(v), \xi\rangle=\frac{1}{2} \omega(\xi \cdot v, v), \quad \text { for any } \quad v \in V, \xi \in \mathfrak{g} .
$$

The symbol $\xi \cdot v$ denotes the infinitesimal generator at $v$ associated to $\xi \in \mathfrak{g}$, and $\langle\cdot, \cdot\rangle$ the natural pairing between the Lie algebra $\mathfrak{g}$ and its dual. Let now $N(K)=\left\{n \in G \mid n K n^{-1}=K\right\}$ be the normalizer of $K$ in $G$. The globally Hamiltonian $G$-action on $V$ induces globally Hamiltonian actions of $L:=N(K) / K$ on $V_{K}$ and $V^{K}$. Moreover, the $L$-action on $V_{K}$ is free. The momentum maps $\mathbf{J}_{L^{K}}: V^{K} \rightarrow \mathfrak{l}^{*}$ and $\mathbf{J}_{L_{K}}: V_{K} \rightarrow \mathfrak{l}^{*}$ associated to these actions are given by

$$
\mathbf{J}_{L^{K}}(v)=\Xi^{*}(\mathbf{J}(v)), \quad \mathbf{J}_{L_{K}}(v)=\Xi^{*}(\mathbf{J}(v)),
$$

where $\Xi^{*}:\left(\mathfrak{k}^{\circ}\right)^{K} \rightarrow \mathfrak{l}^{*}$ is the natural $N(K) / K$-equivariant isomorphism (see [52 55] for the details) between the $K$-fixed points in the annihilator of $\mathfrak{h}$ in $\mathfrak{g}^{*}$ and the dual of the Lie algebra $\mathfrak{l}^{*}$ of $N(K) / K$.

\subsection{The resonance space and normal form reduction}

Let $(V, \omega)$ be a symplectic vector space. It is easy to show that there is a bijection between linear Hamiltonian vector fields on $(V, \omega)$ and quadratic forms on $V$. Indeed, if $A: V \rightarrow V$ is an infinitesimally symplectic linear map, that is, a linear Hamiltonian vector field on $(V, \omega)$, its corresponding Hamiltonian function is given by

$$
Q_{A}(v):=\frac{1}{2} \omega(A v, v), \quad \text { for any } v \in V .
$$

Also, since $A$ belongs to the symplectic Lie algebra $\mathfrak{s p}(V)$, it admits a unique Jordan-Chevalley decomposition [27, 66] of the form $A=A_{s}+A_{n}$, where $A_{s} \in \mathfrak{s p}(V)$ is semisimple (complex diagonalizable), $A_{n} \in \mathfrak{s p}(V)$ is nilpotent, and $\left[A_{s}, A_{n}\right]=0$. If the quadratic form $Q_{A}$ is definite, a theorem of 
Krein [31, 50] guarantees that the associated linear Hamiltonian vector field $A$ is semisimple (complex diagonalizable) and that all its eigenvalues lie on the imaginary axis. Let $i \nu_{\circ}$ be one of the eigenvalues of $A$ and $T_{\nu_{\circ}}:=\frac{2 \pi}{\nu_{0}}$. We define the resonance space $U_{\nu_{\circ}}$ of $A$ with primitive period $T_{\nu_{\circ}}$ as

$$
U_{\nu_{\circ}}:=\operatorname{ker}\left(e^{A_{s} T_{\nu_{\circ}}}-I\right) \text {. }
$$

The resonance space $U_{\nu_{\circ}}$ has the following properties (see 69, 19, 66]):

(i) $U_{\nu_{\circ}}$ is equal to the direct sum of the real generalized eigenspaces of $A$ corresponding to eigenvalues of the form $\pm i k \nu_{0}$, with $k \in \mathbb{N}^{*}$.

(ii) The pair $\left(U_{\nu_{\circ}},\left.\omega\right|_{U_{\nu_{0}}}\right)$ is a symplectic subspace of $(V, \omega)$.

(iii) The mapping $\left.\theta \in S^{1} \mapsto e^{\frac{\theta}{\nu_{0}} A_{s}}\right|_{U_{\nu^{\circ}}}$ generates a symplectic $S^{1}$ linear action on $\left(U_{\nu_{0}},\left.\omega\right|_{U_{\nu_{0}}}\right)$ with associated momentum map $\mathbf{J}_{S^{1}}: U_{\nu_{\circ}} \rightarrow \mathbb{R}$ given by $\mathbf{J}_{S^{1}}=\left.\frac{1}{\nu_{0}} Q_{A_{s}}\right|_{U_{\nu}}$.

(iv) If $(V, \omega)$ is a symplectic representation space of the Lie group $G$ and the Hamiltonian vector field $A$ is $G$-equivariant (equivalently, the quadratic form $Q_{A}$ is $G$-invariant), then the symplectic resonance subspace $\left(U_{\nu_{\circ}},\left.\omega\right|_{U_{\nu_{0}}}\right)$ is also $G$-invariant (this follows from the uniqueness of the JordanChevalley decomposition of $A$, which implies that if $A$ is $G$-equivariant, so is $A_{s}$ ). Moreover, the $S^{1}$ and $G$ actions on $\left(U_{\nu_{0}},\left.\omega\right|_{U_{\nu}}\right)$ commute, which therefore defines a symplectic linear action of $G \times S^{1}$ on $U_{\nu_{0}}$.

(v) The normal form reduction 64, 65, 66] Let $\left(V, \omega, h_{\lambda}\right)$ be a $\lambda$-parameter family $(\lambda \in \Lambda$, where $\Lambda$ is a Banach space) of smooth $G$-Hamiltonian systems such that for any $\lambda \in \Lambda, h_{\lambda}(0)=0$, $\mathbf{d} h_{\lambda}(0)=0$, and the $G$-equivariant infinitesimally symplectic linear map $A:=D X_{h_{\lambda_{0}}}(0)$ is non singular and has $\pm i \nu_{\circ}$ as eigenvalues. Let $\left(U_{\nu_{0}},\left.\omega\right|_{U_{\nu_{0}}}\right)$ be the resonance space of $A$ with primitive period $T_{\nu_{\circ}}$. Then, there exist smooth mappings $\psi: U_{\nu_{\circ}} \times \Lambda \rightarrow V$ and $C^{k+1}$-mapping $\widehat{h_{\lambda}}$ : $U_{\nu_{\circ}} \times \Lambda \rightarrow \mathbb{R}$ such that $\psi(0, \lambda)=0$, for all $\lambda \in \Lambda, D_{U_{\nu_{0}}} \psi\left(0, \lambda_{\circ}\right)=\mathbb{I}_{U_{\nu_{\circ}}}\left(D_{U_{\nu}}\right.$ denotes the partial Fréchet derivative relative to the variable in $U_{\nu_{0}}$ ), and, most importantly [65, Theorem V.5.17], 66, Theorem 3.2], if we stay close enough to zero in $U_{\nu_{\circ}}$ and to $\lambda_{\circ} \in \Lambda$, then the $S^{1}$-relative equilibria of the $G \times S^{1}$-invariant Hamiltonian $\widehat{h_{\lambda}}$ are mapped by $\psi(\cdot, \lambda)$ to the set of periodic solutions of $\left(V, \omega, h_{\lambda}\right)$ in a neighborhood of $0 \in V$, with periods close to $T_{\nu_{\circ}}$. Hence, in our future discussion we will substitute the problem of searching periodic orbits for $\left(V, \omega, h_{\lambda}\right)$ by that of searching the $S^{1}$-relative equilibria of the $G \times S^{1}$-invariant family of Hamiltonian systems $\left(U_{\nu_{\circ}},\left.\omega\right|_{U_{\nu_{\circ}}}, \widehat{h_{\lambda}}\right)$, that will be referred to as the equivalent system. Given any $k \in \mathbb{N}$, the equivalent system Hamiltonian $\widehat{h_{\lambda}}$ can be chosen so that its Taylor expansion coincides with that of $\left.h_{\lambda}\right|_{U_{\nu}}$ up to order $k$. This fact and the properties of $\psi$ imply that

$$
\mathcal{A}:=\left.A\right|_{U_{\nu^{\circ}}}=\left.D_{V} X_{h_{\lambda_{0}}}(0)\right|_{U_{\nu_{0}}}=D_{U_{\nu_{0}}} X_{\left.h_{\lambda_{0}}\right|_{U_{\nu_{0}}}}(0)=D_{U_{\nu_{0}}} X_{\widehat{h_{\lambda_{0}}}}(0) .
$$

The reduction procedure that we just described appears also in the literature under the name of Weinstein-Moser reduction.

\subsection{Hamiltonian relative equilibria and relative periodic orbits}

A point $m \in M$ is a relative equilibrium of the Hamiltonian system with symmetry $(M, \omega, h, G, \mathbf{J})$, with velocity $\xi \in \mathfrak{g}$, iff $m$ is a critical point of the augmented Hamiltonian $h^{\xi}:=h-\mathbf{J}^{\xi}$, where $\mathbf{J}^{\xi}:=\langle\mathbf{J}, \xi\rangle$. A similar characterization for RPOs that also uses the momentum map is given in the following elementary result.

Proposition 2.1 Let $(M, \omega, h)$ be a Hamiltonian system with a globally Hamiltonian symmetry given by the canonical action of the Lie group $G$ on $M$, with associated momentum map $\mathbf{J}: M \rightarrow \mathfrak{g}^{*}$. If the Hamiltonian vector field $X_{h-\mathbf{J}} \xi, \xi \in \mathfrak{h}$, has a periodic point $m \in M$ with period $\tau$, then the point $m$ is a RPO of $X_{h}$ with relative period $\tau$ and phase shift $\exp \tau \xi$. 
Proof Let $F_{t}$ be the flow of the Hamiltonian vector field $X_{h}$ and $K_{t}(m)=\exp t \xi \cdot m$ that of $X_{\mathbf{J}} \xi$. By Noether's Theorem we have that $\left[X_{h}, X_{\mathbf{J}^{\xi}}\right]=-X_{\left\{h, \mathbf{J}^{\xi}\right\}}=0$, where the bracket $\{\cdot, \cdot \cdot$ denotes the Poisson bracket associated to the symplectic form $\omega$. Due to this equality, we can write (see for instance [2] Corollary 4.1.27]) the following expression for $G_{t}$, the flow of $X_{h-\mathbf{J}^{\xi}}, G_{t}(m)=\lim _{n \rightarrow \infty}\left(F_{t / n} \circ K_{-t / n}\right)^{n}(m)=$ $\left(K_{-t} \circ F_{t}\right)(m)=\exp -t \xi \cdot F_{t}(m)$. Since by hypothesis the point $m$ is periodic for $G_{t}$ with period $\tau$, we have that $m=\exp -\tau \xi \cdot F_{\tau}(m)$, or, equivalently, $F_{\tau}(m)=\exp \tau \xi \cdot m$, as required.

The proposition that we just proved allows us to search the RPOs of the system $(M, \omega, h, G, \mathbf{J})$ by looking at the periodic orbits of the systems with Hamiltonian functions the augmented Hamiltonians $h^{\xi}:=h-\mathbf{J}^{\xi}$. Notice that in terms of symmetry properties, the new systems whose periodic orbits we want to compute are weaker. More specifically, even though the original Hamiltonian is $G$-invariant, the augmented Hamiltonian $h-\mathbf{J}^{\xi}$ is only $G^{\xi}$-invariant, where $G^{\xi}$ is the adjoint isotropy of the element $\xi \in \mathfrak{g}$, that is, $G^{\xi}:=\left\{g \in G \mid \operatorname{Ad}_{g} \xi=\xi\right\}$.

As we already mentioned in the introduction, the use of the previous proposition in the search for the RPOs of a system carries intrinsically two main limitations. Firstly, since the phase shift of a RPO that amounts to a periodic orbit of $h-\mathbf{J}^{\xi}$ is always of the form $\exp \tau \xi$, with $\tau$ some real number. Hence, the RPOs whose phase shifts do not lie in the connected component of the identity of the group of symmetries $G$ cannot possibly be found in this way. Second, if $G$ is a discrete group then its Lie algebra is trivial and consequently so is the momentum map associated to this action, which makes the previous proposition empty of content.

\subsection{Results on critical point theory of functions on compact manifolds}

\subsubsection{The Lusternik-Schnirelman approach}

The following two results are slight generalizations of those presented in 68 for circle actions. The additional hypotheses that we will introduce in our statements will make the original proofs work with straightforward modifications.

Proposition 2.2 Let $M$ be a compact $G$-manifold, with $G$ a Lie group acting properly on $M$. Any $G$-invariant smooth function $f \in C^{\infty}(M)^{G}$ has at least

$$
\operatorname{Cat}(M / G)
$$

critical orbits.

In the previous statement, the symbol Cat denotes the Lusternik-Schnirelman category of the quotient compact topological space $M / G$ (the action of $G$ on $M$ does not need to be free and, consequently, the quotient $M / G$ is not in general a manifold). Recall that the Lusternik-Schnirelman category of a compact topological space $M$ is the minimal number of closed contractible sets needed to cover $M$.

The preceding statement is one of the main analytical tools that we will use to obtain estimates on the number of RPOs of our problem. As we will see later on, we will be able to reduce the search for those RPOs to the computation of the number of critical orbits of a $G$-invariant function defined on a compact $G$-symmetric manifold that satisfies the hypotheses of Proposition 2.2 In principle, the category in (2.2) is very difficult to compute. Nevertheless, in our situation we will take advantage of the symplectic nature of our setup to estimate it in terms of readily computable dimensional quantities. The main tools to carry that out are the following technical propositions whose relevance will become apparent at the time of their application in the proof of the main theorems.

Proposition 2.3 Let $M$ be a compact $G$-manifold, with $G$ a Lie group acting properly on $M$ such that the isotropy subgroup of each point $m \in M$ is a finite subgroup of $G$. Let $\omega$ be a symplectic $G$-invariant form defined on $M$. Let $H \subset G$ be a Lie subgroup of $G$ and $N \subset M$ be a $H$-invariant closed submanifold of $M$ such that for any $n \in N$ we have that

$$
\left(T_{n} N\right)^{\omega}=\mathfrak{g} \cdot n \quad \text { and } \quad T_{n} N \cap \mathfrak{g} \cdot m=\mathfrak{h} \cdot n,
$$


where $\mathfrak{g} \cdot n=\left\{\xi_{M}(n):=\left.\frac{d}{d t}\right|_{t=0} \exp t \xi \cdot n \mid \xi \in \mathfrak{g}\right\}$ denotes the tangent space at $n \in N \subset M$ of the $G$-orbit $G \cdot n=\{g \cdot n \mid g \in G\}$. Then, there is a cohomology class $\theta \in H^{2}(N / H ; \mathbb{R})$ such that $\theta^{k} \neq 0$, where $k=\frac{1}{2}(\operatorname{dim} N-\operatorname{dim} H)$.

The proof of the following elementary fact can be found in 61 .

Proposition 2.4 Let $M$ be a compact topological space. The Lusternik-Schnirelman category of $M$ is at least 1 plus its cuplength.

Corollary 2.5 Let $(M, \omega)$ be a $2 n$-dimensional compact symplectic manifold. Then,

$$
\operatorname{Cat}(M) \geq 1+n \text {. }
$$

Proof The symplecticity of $\omega$ implies that $\omega^{n}$ is a nowhere vanishing multiple of the volume form, hence $[\omega]^{n}=\left[\omega^{n}\right] \neq 0$ in the top cohomology group of the manifold, and therefore the cuplength of the manifold $M$ is at least $n$. The conclusion follows from Proposition 2.4

Another approach to the search of critical orbits of symmetric functions is the use of the so called equivariant Lusternik-Schnirelman category or G-Lusternik-Schnirelman category (denoted by the symbol $G$-Cat), introduced in different versions and degrees of generality by Fadell [18, Clapp and Puppe [12, 13, and Marzantowicz [44. The equivariant Lusternik-Schnirelman category is not the standard Lusternik-Schnirelman category of the orbit space that we used in the previous paragraphs, but the minimal cardinality of a covering of the $G$-manifold $M$ by $G$-invariant closed subsets that can be equivariantly deformed to an orbit. This new category is also a lower bound for the number of critical orbits of a $G$-invariant function on $M$ and is actually a better bound since it can be proven (see for instance [18, page 43]) that $G-\operatorname{Cat}(M) \geq \operatorname{Cat}(M / G)$, where the equality holds, for instance, when the $G$-action on $M$ is free. Nevertheless, we will not use this category since the cohomological estimates that can be made via arguments similar to those established using propositions 2.3 and 2.4 on the value of the $G$-category require the use of $G$-equivariant cohomology hence giving rise to estimates that are not as readily computable as those that we will obtain using standard cohomology.

\subsubsection{The Morse theoretical approach}

The following paragraphs briefly summarize some results on Morse Theory adapted to the symmetric Hamiltonian setup as they can be read in the works of Kirwan 29, 30.

Let $f \in C^{\infty}(M)$ be a smooth function on the compact manifold $M$. A critical point $m \in M$ is said to be non-degenerate when the second derivative of $f$ at the critical point $\mathbf{d}^{2} f(m)$ is a non degenerate quadratic form. Non degenerate critical points are isolated. The index $i_{f}(m)$ of a critical point $m$ is the maximal dimension of a subspace of $T_{m} M$ in which $\mathbf{d}^{2} f(m)$ is negative definite. A smooth function all whose critical points are non degenerate is said to be of Morse type.

If $M$ is a $G$-manifold with $G$ a Lie group of positive dimension, the critical points of any smooth $G$-invariant function $f \in C^{\infty}(M)^{G}$ come in $G$-orbits. Therefore, since the critical points of $f$ are not isolated the function $f$ cannot possibly be of Morse type. The closest condition to being of Morse type that we can envision in the equivariant context is what we will call being of $G$-Morse. A function $f \in C^{\infty}(M)^{G}$ is of $G$-Morse when all its critical points $m \in M$ satisfy that

$$
\operatorname{ker} \mathbf{d}^{2} f(m)=T_{m}(G \cdot m) .
$$

This condition will be needed in the statement of our main results. Note that this is a "reasonable" condition to be imposed since it is generic in the $G$-category. A situation in which it is very easy to see that this is the case is when the $G$-action on $M$ is free. In that case, a $G$-invariant function being $G$-Morse is equivalent to its projection onto the quotient manifold $M / G$ being a standard Morse function, which occurs generically.

The $G$-Morse condition implies the minimal degeneracy condition [29], Appendix 10], which is the weakest condition that, to the knowledge of the author, allows the formulation of Morse inequalities and 
standard Morse theory (see [5, 45] for additional information). More specifically, the Morse inequalities in this situation state that if the function $f \in C^{\infty}(M)^{G}$ is $G$-Morse then there exits a polynomial $R(t)$ in $t$ with non negative integer coefficients such that

$$
\sum_{G \cdot x \text { critical orbit of } f} t^{i_{f}(G \cdot x)} P_{t}(G \cdot x)-P_{t}(M)=R(t)(1+t), \quad R(t) \geq 0,
$$

where the sum runs over all the critical orbits $G \cdot x$ of the function $f$, and $P_{t}(G \cdot x), P_{t}(M)$ denote the Poincaré series of $G \cdot x$ and $M$ respectively

$$
P_{t}(G \cdot x)=\sum_{i \geq 0} b_{i}(G \cdot x) t^{i}, \quad P_{t}(M)=\sum_{i \geq 0} b_{i}(M) t^{i} .
$$

The symbols $b_{i}(M)$ (resp. $\left.b_{i}(G \cdot x)\right)$ denote the Betti numbers of the manifold $M$ (resp. $\left.G \cdot x\right)$, that is,

$$
b_{i}(M)=\operatorname{dim} H_{i}(M) \quad\left(\text { resp. } b_{i}(G \cdot x)=\operatorname{dim}(G \cdot x)\right) .
$$

The Morse inequalities (2.5) still hold if instead of using ordinary cohomology we use equivariant cohomology, that is, there exits a polynomial $R(t)$ in $t$ with non negative integer coefficients such that

$$
\sum_{G \cdot x \text { critical orbit of } f} t^{i_{f}(G \cdot x)}-P_{t}^{G}(M)=R(t)(1+t), \quad R(t) \geq 0,
$$

where $P_{t}^{G}(M)=\sum_{i>0} t^{i} \operatorname{dim} H_{G}^{i}(M)$. These are the so called equivariant Morse inequalities.

A straightforward consequence of (2.6) is that any $G$-invariant function $f$ on the compact manifold $M$ has at least

$$
\left|\chi(M)^{G}\right|:=\left|P_{-1}^{G}(M)\right|
$$

critical orbits. The number $\chi(M)^{G}$ is called the $G$-Euler characteristic of $M$.

The previous remark is particularly relevant in the globally Hamiltonian framework: suppose now that the compact $G$-manifold $M$ is symplectic, that the Lie group $G$ acts canonically, and that this action has an equivariant momentum map associated $\mathbf{J}: M \rightarrow \mathfrak{g}^{*}$. Let $\mu \in \mathbf{J}(M) \subset \mathfrak{g}^{*}$ be a regular value of $\mathbf{J}$ and $G_{\mu}$ be the corresponding coadjoint isotropy subgroup which we will assume acts in a locally free fashion on $\mathbf{J}^{-1}(\mu)$. In the proofs of our main results it will be necessary to evaluate the number of critical orbits of a $G_{\mu}$-invariant function on $\mathbf{J}^{-1}(\mu)$, which by (2.7) can be done just by computing $\chi\left(\mathbf{J}^{-1}(\mu)\right)^{G_{\mu}}$. This work has been carried out in full detail by Kirwan 29, 30] who realized that the function $f_{\mu}:=\|\mathbf{J}-\mu\|^{2} \in C^{\infty}(M)^{G_{\mu}}\left(\|\cdot\|\right.$ is any $\operatorname{Ad}_{G}^{*}$-invariant inner product on $\left.\mathfrak{g}^{*}\right)$ is an equivariantly perfect Morse function, which allows to explicitly write down (see 30. Theorem 4.14]) the $G_{\mu}$-equivariant Betti numbers of $\mathbf{J}^{-1}(\mu)$, and consequently $\chi\left(\mathbf{J}^{-1}(\mu)\right)^{G_{\mu}}$, in terms of the Betti numbers of $M$, of the classifying space of $G_{\mu}$, and of the equivariant Betti numbers of some simpler subspaces of $M$ explicitly constructed using the geometry of the momentum map. Therefore, in our future discussions we will consider $\chi\left(\mathbf{J}^{-1}(\mu)\right)^{G_{\mu}}$ as a computable quantity that we can use, via (2.7), in our estimations on the number of $G_{\mu}$-critical orbits on $\mathbf{J}^{-1}(\mu)$ (see Section 3 in 30 for an explicit example of this calculation).

We conclude this remark by noting that since we will be working with locally free actions, the rational equivariant cohomology ring $H_{G_{\mu}}^{*}\left(\mathbf{J}^{-1}(\mu) ; \mathbb{Q}\right)$ is isomorphic to the ordinary rational cohomology ring $H^{*}\left(\mathbf{J}^{-1}(\mu) / G_{\mu} ; \mathbb{Q}\right)$, and therefore

$$
\chi\left(\mathbf{J}^{-1}(\mu)\right)^{G_{\mu}}=\chi\left(\mathbf{J}^{-1}(\mu) / G_{\mu}\right) .
$$

\subsection{Lagrange multipliers}

The use of Lagrange multipliers will be crucial in the proof techniques that we will use. The version of this result that we present in the following proposition can be found as Corollary 3.5.29 in [2].

Proposition 2.6 Let $M$ be a smooth manifold, $F$ be a Banach space, $g: M \rightarrow F$ be a smooth submersion, $f \in C^{\infty}(\mathbb{R})$, and $N=g^{-1}(0)$. The point $n \in N$ is a critical point of the restriction $\left.f\right|_{N}$ iff there exists $\lambda \in F^{*}$, called a Lagrange multiplier, such that $n$ is a critical point of $f-\lambda \circ g$. 


\section{The main result}

\subsection{Relative periodic orbits with prescribed isotropy}

Before we state the main result of this section we need some terminology. Let $h \in C^{\infty}(V)$ a smooth function defined on the vector space $V$ such that $h(0)=0, \mathbf{d} h(0)=0$, and the second derivative at zero $Q:=\mathbf{d}^{2} h(0)$ is a definite quadratic form. Let $\langle\cdot, \cdot\rangle$ be the scalar product on $V$ defined by

$$
\langle u, v\rangle:=\frac{1}{2} \mathbf{d}^{2} h(0)(u, v), \quad u, v \in V,
$$

and $\|\cdot\|$ be the associated norm. We will say that these are the scalar product and the norm associated to the quadratic form $Q$. We now write the Taylor expansion of $h$ around the origin:

$$
h(v)=\|v\|^{2}+\frac{1}{3} \mathbf{d}^{3} h(0)\left(v^{(3)}\right)+\cdots+\frac{1}{k !} \mathbf{d}^{k} h(0)\left(v^{(k)}\right)+\cdots \quad v \in V .
$$

We will say that the $k$ th term in the Taylor expansion of the function $h$ is purely radial when

$$
\frac{1}{k !} \mathbf{d}^{k} h(0)\left(v^{(k)}\right)=c_{k}\|v\|^{k}
$$

where $c_{k}$ is a constant real number.

The main goal of this section is proving the following theorem:

Theorem 3.1 Let $\left(V, \omega, h, G, \mathbf{J}: V \rightarrow \mathfrak{g}^{*}\right)$ be a Hamiltonian system with symmetry, with $V$ a vector space, and $G$ a compact positive dimensional Lie group that acts on $V$ in a linear and canonical fashion. Suppose that $h(0)=0, \mathbf{d} h(0)=0$ (that is, the Hamiltonian vector field $X_{h}$ has an equilibrium at the origin) and that the linear Hamiltonian vector field $A:=D X_{h}(0)$ is non degenerate and contains $\pm i \nu_{\circ}$ in its spectrum. Let $U_{\nu_{\circ}}$ be the resonance space of $A$ with primitive period $T_{\nu_{\circ}}:=\frac{2 \pi}{\nu_{0}}$. Let $K \subset G$ be an isotropy subgroup of the $G$-action on $V$ for which the quadratic form $Q^{K}$ on the $K$-fixed point space $U_{\nu_{\circ}}^{K}$ defined by

$$
Q^{K}(v):=\frac{1}{2} \mathbf{d}^{2} h(0)(v, v), \quad v \in U_{\nu_{\circ}}^{K}
$$

is definite, and the set $\mathbf{J}_{L_{K}}^{-1}(0) \cap Q_{K}^{-1}(1)$ is non empty $\left(Q_{K}:=\left.Q^{K}\right|_{\left(U_{\nu_{0}}\right)_{K}}\right)$. In these conditions, there exists a neighborhood $B(K) \subset \mathfrak{l}^{*}$ of zero in $\mathfrak{l}^{*}$ such that for any $\lambda \in B(K)$, the intersection $\mathbf{J}_{L_{K}}^{-1}(\lambda) \cap$ $Q_{K}^{-1}(1)$ is a submanifold of $\left(U_{\nu_{\circ}}\right)_{K}$. Suppose that the following two generic hypotheses hold:

(H1) The restriction $\left.h\right|_{U_{\nu_{0}^{K}}^{K}}$ of the Hamiltonian $h$ to the fixed point subspace $U_{\nu_{\circ}}^{K}$ is not radial with respect to the norm associated to $Q^{K}$.

(H2) Let $h_{k}(v):=\frac{1}{k !} \mathbf{d}^{k} h(0)\left(v^{(k)}\right), v \in U_{\nu_{\circ}}^{K}$ be the first non radial term in the Taylor expansion of $h_{U_{\nu_{0}}^{K}}$ around zero. We will assume that $k \geq 4$ and that the restrictions $\left.h_{k}\right|_{\mathbf{J}_{L_{K}}^{-1}(\lambda) \cap Q_{K}^{-1}(1)}$ of $h_{k}$ to the submanifolds $\mathbf{J}_{L_{K}}^{-1}(\lambda) \cap Q_{K}^{-1}(1)$, with $\lambda \in B(K)$, are $(N(K) / K)_{\lambda} \times S^{1}-$ Morse.

Then, the neighborhood $B(K)$ can be chosen so that for any $\epsilon>0$ close enough to zero and any $\lambda \in B(K)$ there are at least

$$
\max \left[\frac{1}{2}\left(\operatorname{dim} U_{\nu_{\circ}}^{K}-\operatorname{dim}(N(K) / K)-\operatorname{dim}(N(K) / K)_{\lambda}\right), \chi\left(\mathbf{J}_{L_{K}}^{-1}(\lambda) \cap Q_{K}^{-1}(1)\right)^{L_{\lambda} \times S^{1}}\right]
$$

distinct relative periodic orbits of $X_{h}$ with energy $\epsilon$, momentum $\epsilon\left(\Xi_{K}^{*}\right)^{-1}(\lambda) \in \mathfrak{g}^{*}$, isotropy subgroup $K$, and relative period close to $T_{\nu_{0}}$. The choice of $B(K)$ guarantees that these relative periodic orbits are not just relative equilibria. The symbol $N(K)$ denotes the normalizer of $K$ in $G, \mathbf{J}_{L_{K}}:\left(U_{\nu_{\circ}}\right)_{K} \rightarrow \mathfrak{l}^{*}$ is the momentum map associated to the free $L:=N(K) / K$-action on $\left(U_{\nu_{0}}\right)_{K}, \Xi_{K}^{*}$ denotes the natural isomorphism $\Xi_{K}^{*}:\left(\mathfrak{h}^{\circ}\right)^{K} \rightarrow \mathfrak{l}^{*}$, and $(N(K) / K)_{\lambda}$ the coadjoint isotropy of $\lambda \in \mathfrak{l}^{*}$ (see Section 2.1] for 
more information about this notation). The symbol $\chi\left(\mathbf{J}_{L_{K}}^{-1}(\lambda) \cap Q_{K}^{-1}(1)\right)^{L_{\lambda} \times S^{1}}$ denotes the $L_{\lambda} \times S^{1}-$ Euler characteristic of $\mathbf{J}_{L_{K}}^{-1}(\lambda) \cap Q_{K}^{-1}(1)$ (which in this case equals the standard Euler characteristic of the symplectic quotient $\left.\chi\left(\mathbf{J}_{L_{K}}^{-1}(\lambda) \cap Q_{K}^{-1}(1) / L_{\lambda} \times S^{1}\right)\right)$.

Remark 3.2 The main estimate (3.1) contains two parts. The first one, in terms of various dimensions, is in general easy to compute (see the examples below) and has been obtained via a cohomological estimate on the Lusternik-Schnirelmann category of certain symplectic orbifold. The second part involving the Euler characteristic is in general a sharper estimate but also much more difficult to compute. The reader is referred to [30] for an example on how to compute these quantities.

Remark 3.3 As we will see in the proof, the properties of the non trivial $S^{1}$-action on the space $U_{\nu_{\circ}}^{K}$ (normal form) imply that the number $k$ in the statement of hypothesis (H2) is necessarily even.

Examples 3.4 We illustrate with a few elementary examples the use of Theorem 3.1 in specific situations.

(i) Nonlinearly perturbed spherical pendulum: the spherical pendulum consists of a particle of mass $m$, moving under the action of a constant gravitational field of acceleration $g$, on the surface of a sphere of radius $l$. We consider this system perturbed by an $S^{1}$-invariant nonlinear term. If we use as local coordinates of the configuration space around the downright position the Cartesian coordinates $(x, y)$ of the orthogonal the projection of the sphere on the equatorial plane, the (local) Hamiltonian of this system is:

$$
h\left(x, y, p_{x}, p_{y}\right)=\frac{p_{x}^{2}}{2 m}+\frac{p_{y}^{2}}{2 m}-\frac{\left(x p_{x}+y p_{y}\right)^{2}}{2 m l^{2}}-m g \sqrt{l^{2}-x^{2}-y^{2}}+\varphi\left(x^{2}+y^{2}, p_{x}^{2}+p_{y}^{2}, x p_{x}+y p_{y}\right),
$$

where the function $\varphi$ is of order two or higher in all of its variables and encodes the nonlinear perturbation. This system is invariant with respect to the globally Hamiltonian $S^{1}$-action given by the expression $\Phi_{\theta}\left(x, y, p_{x}, p_{y}\right)=\left(R_{\theta}(x, y), R_{\theta}\left(p_{x}, p_{y}\right)\right)$, where $R_{\theta}$ denotes a rotation of angle $\theta$. The momentum map $\mathbf{J}: \mathbb{R}^{4} \rightarrow \mathbb{R}$ associated to this action is given by $\mathbf{J}\left(x, y, p_{x}, p_{y}\right)=x p_{y}-y p_{x}$. The point $\left(x, y, p_{x}, p_{y}\right)=(0,0,0,0)$ is an stable equilibrium of the Hamiltonian vector field $X_{h}$ to which we apply Theorem 3.1] Indeed, the linearization of $X_{h}$ at the origin has two imaginary eigenvalues that are forced to be double by the symmetry of the problem. Consequently, the associated resonance space coincides with the entire $\mathbb{R}^{4}$. We now use the dimensional estimate in (3.1) to look for RPOs with trivial isotropy. A straightforward dimension count shows that when $K=\{e\}$, the orbifold $\mathbf{J}^{-1}(0) \cap Q_{e}^{-1}(1) / S^{1} \times S^{1}$ is zero dimensional and therefore hypotheses (H1) and (H2) trivially hold for the standard spherical pendulum $(\varphi \equiv 0)$ and for ALL non radial perturbations $\varphi$. Therefore, for any value of the momentum and the energy neighboring 0 , there exists at least one $\boldsymbol{R P O}$ with trivial isotropy. An in-depth study of these motions can be found in [14.

(ii) The spring pendulum in three dimensions: the spring pendulum in space is a bob of mass $m$ under the action of a constant gravitational field of acceleration $g$ attached to a spring whose length at rest equals $l$. The Hamiltonian function associated to this system is

$$
h\left(x, y, z, p_{x}, p_{y}, p_{z}\right)=\frac{1}{2}\left(p_{x}^{2}+p_{y}^{2}+p_{z}^{2}\right)-m g z+\sigma\left(x^{2}+y^{2}+(z-l)^{2}, p_{x}^{2}+p_{y}^{2}, z, p_{z}\right) .
$$

where $\sigma$ is a smooth real valued function such that $\sigma\left(x^{2}+y^{2}+(z-l)^{2}, p_{x}^{2}+p_{y}^{2}, z, p_{z}\right)=\frac{k}{2}\left(x^{2}+\right.$ $\left.y^{2}+(z-l)^{2}\right)+$ terms of order higher or equal than two in the first two variables and strictly higher than two in $z$ and $p_{z}$. This system presents the same $S^{1}$-symmetry as the previous example. The point $\left(0,0, l+\frac{g m}{k}, 0,0,0\right)$ is a stable equilibrium where the linearization of the Hamiltonian vector field has two pairs of triple purely imaginary eigenvalues equal to $\pm \frac{i}{2} \sqrt{\frac{k}{m}}$. Hence, in this case, the resonance space is six dimensional. When there are no higher order terms in the expansion of the 
function $\sigma$ we have a linear spring (which does not mean that its associated Hamiltonian vector field is linear) obeying Hooke's law with elastic constant $k$. In this case, the spring pendulum is too degenerate and does not satisfy hypothesis (H2). However, in the presence of generic non linear terms in the expansion of $\sigma$, Theorem 3.1 predicts the existence of $\boldsymbol{t w o} \boldsymbol{R P O s}$ with trivial isotropy, for any value of the momentum and the energy neighboring the values at the equilibrium.

(iii) An example with spherical symmetry: consider $T^{*} \mathbb{R}^{3} \simeq \mathbb{R}^{3} \times \mathbb{R}^{3}$ with the canonical symplectic form and the group $\mathrm{SO}(3)$ acting canonically by diagonal transformations. If we denote by $(\mathbf{q}, \mathbf{p})$ the elements of $\mathbb{R}^{3} \times \mathbb{R}^{3}$, the momentum map associated to this action is the standard angular momentum $\mathbf{J}(\mathbf{q}, \mathbf{p})=\mathbf{q} \times \mathbf{p}$. Consider the Hamiltonian function:

$$
h(\mathbf{q}, \mathbf{p})=a\|\mathbf{p}\|^{2}+b\|\mathbf{q}\|^{2}+f\left(\|\mathbf{p}\|^{2},\|\mathbf{q}\|^{2}, \mathbf{q} \cdot \mathbf{p}\right),
$$

where $a$ and $b$ are real strictly positive constants and $f$ is a function whose Taylor expansion only contains terms of order two or higher in its variables. The associated Hamiltonian vector field has a stable equilibrium at the origin and its linearization at that point exhibits one pair of triple purely imaginary eigenvalues that therefore have a six dimensional resonance space associated. We now look for RPOs nearby the equilibrium. The origin is surrounded by points with isotropy subgroup $K$ either trivial or equal to a circle. When $K=S^{1}$, the vectors $\mathbf{q}$ and $\mathbf{p}$ are forced to be parallel and therefore its momentum value equal to zero, hence there is only room for planar periodic solutions. When $K=\{e\}$, the vectors $\mathbf{q}$ and $\mathbf{p}$ are necessarily not parallel and therefore its angular momentum is different from zero. Now, for any momentum value $\mu \in \mathbb{R}^{3}$ close to zero, the orbifold $\mathbf{J}^{-1}(\mu) \cap Q_{\{e\}}^{-1}(1) /\left((\mathrm{SO}(3))_{\mu} \times S^{1}\right)$ is zero dimensional because $(\mathrm{SO}(3))_{\mu} \simeq S^{1}$ consequently, any function $f$ that depends non-trivially on $\mathbf{q} \cdot \mathbf{p}$ makes the Hamiltonian vector field associated to the corresponding $h$ exhibit, by Theorem [3.1] at least one $\boldsymbol{R P O}$ with trivial isotropy, for any value of the momentum and the energy neighboring the values at the equilibrium.

Remark 3.5 Note that in the absence of symmetries and for non trivial manifolds, that is $G=\{e\}$ and $\operatorname{dim} V>0$, the first part of the main estimate (3.1) reduces to

$$
\frac{1}{2}\left(\operatorname{dim} U_{\nu_{\circ}}\right)
$$

which coincides with the conclusions of Moser's version [51] of Theorem 1.1

Remark 3.6 The main feature of the first part of the estimate (3.1), namely,

$$
\frac{1}{2}\left(\operatorname{dim} U_{\nu_{\circ}}^{K}-\operatorname{dim}(N(K) / K)-\operatorname{dim}(N(K) / K)_{\lambda}\right)
$$

is that it does not involve any dynamics, that is, neither the Hamiltonian $h$ nor any of its byproducts are present in it. The kinematical setup of the problem, in our case given by the symplectic representation of $G$ on $V$, fully determine the number of RPOs that can be expected around a stable symmetric equilibrium, induced by ANY Hamiltonian system that satisfies the hypotheses of the Theorem.

This dynamical independence, that was already present in the non symmetric result of Weinstein and Moser (3.2), has a price in terms of sharpness, since in general, the Morse theoretical part of (3.1) involving the Euler characteristic is expected to give better results.

Remark 3.7 Despite the one half in front of the first part of estimate (3.1) we always obtain an integer out of it. Indeed, $U_{\nu_{\circ}}^{K}$ is a symplectic vector subspace of the symplectic vector space $U_{\nu_{\circ}}$, and therefore of even dimension. Also, the coadjoint orbit $(N(K) / K) \cdot \lambda$ is also a symplectic manifold of even dimension equal to $\operatorname{dim}(N(K) / K)-\operatorname{dim}(N(K) / K)_{\lambda}$, hence $-\operatorname{dim}(N(K) / K)-\operatorname{dim}(N(K) / K)_{\lambda}$ is necessarily also an even number. 


\subsection{Proof of Theorem 3.1}

The proof of Theorem 3.1 is unfortunately quite long and technical. In order to make it more accessible to the reader I have divided it into several subsections that accomplish a self-contained task.

The general strategy of the proof consists of characterizing the relative periodic orbits that we are looking for, through the use of the normal form theorem, as the critical points of an equivariant function on certain manifold. The orbit space of this manifold with respect to the natural group action that leaves it invariant is a symplectic orbifold (the group action is locally free) which allows us to implement the various critical point theory techniques that we introduced in Section 2.4

\subsubsection{The manifold $\mathbf{J}_{L_{K}}^{-1}(\lambda) \cap Q^{-1}(1)$}

We start the proof of the theorem by showing that for momentum values $\lambda \in \mathfrak{l}^{*}$ nearby zero, the manifolds $\mathbf{J}_{L_{K}}^{-1}(\lambda)$ and $Q^{-1}(1)$ intersect transversely and, therefore, the intersection forms a manifold. This manifold is important because it will constitute the numerator of the symplectic orbifold that we described in the previous paragraph.

Proposition 3.8 Suppose that we are under the hypotheses of Theorem 3.1. Let $K \subset G$ be an isotropy subgroup of the $G$-action on $U_{\nu_{\circ}}$ and $\mathbf{J}_{L_{K}}:\left(U_{\nu_{\circ}}\right)_{K} \rightarrow \mathfrak{I}^{*}$ be the momentum map corresponding to the free $N(K) / K$-action on $\left(U_{\nu_{\mathrm{o}}}\right)_{K}$. Suppose that the set $\mathbf{J}_{L_{K}}^{-1}(0) \cap Q_{K}^{-1}(1)$ is not empty. Then, there is a neighborhood $B(K) \subset \mathfrak{l}^{*}$ of 0 in $\mathfrak{l}^{*}$ such that for any $\lambda \in B(K)$ :

(i) $\mathbf{J}_{L_{K}}^{-1}(\lambda) \pitchfork Q^{-1}(1)=\mathbf{J}_{L_{K}}^{-1}(\lambda) \pitchfork Q_{K}^{-1}(1)$, that is, $\mathbf{J}_{L_{K}}^{-1}(\lambda)$ intersect transversely with $Q^{-1}(1)$ and with $Q_{K}^{-1}(1)$.

(ii) $\mathbf{J}_{L_{K}}^{-1}(\lambda) \cap Q^{-1}(1)=\mathbf{J}_{L_{K}}^{-1}(\lambda) \cap Q_{K}^{-1}(1)$ is a compact submanifold of $\left(U_{\nu_{\circ}}\right)_{K}$ of dimension $\operatorname{dim}\left(U_{\nu_{\circ}}\right)_{K}-$ $\operatorname{dim} N(K) / K-1=\operatorname{dim} U_{\nu_{\circ}}^{K}-\operatorname{dim} N(K) / K-1$.

(iii) The submanifold $\mathbf{J}_{L_{K}}^{-1}(\lambda) \cap Q^{-1}(1)=\mathbf{J}_{L_{K}}^{-1}(\lambda) \cap Q_{K}^{-1}(1) \subset\left(U_{\nu_{0}}\right)_{K}$ does not contain any $N(K) / K-$ relative equilibrium of the system $\left(\left(U_{\nu_{\circ}}\right)_{K},\left.\omega\right|_{\left(U_{\nu}\right)_{K}}, Q_{K}\right)$.

Proof (i) Given that the $N(K) / K$-action on $\left(U_{\nu_{\mathrm{o}}}\right)_{K}$ is free, the momentum map $\mathbf{J}_{L_{K}}$ is a submersion and therefore its level sets are always submanifolds of $\left(U_{\nu_{\circ}}\right)_{K}$, and consequently of $U_{\nu_{\circ}}$. At the same time, the definiteness of the quadratic form $Q$ implies that its level sets are compact submanifolds of $U_{\nu_{0}}$. We will show the transversality of the level sets in the statement by showing that $\mathbf{J}_{L_{K}}^{-1}(0) \pitchfork$ $Q^{-1}(1)$. Since the transversality is an open condition (see for instance the Stability Theorem in page 35 of [22]), the result will follow for $\mathbf{J}_{L_{K}}^{-1}(\lambda) \pitchfork Q^{-1}(1)$, for $\lambda$ close enough to zero. In order to prove that $\mathbf{J}_{L_{K}}^{-1}(0) \pitchfork Q^{-1}(1)$ we need to show that for each $v \in \mathbf{J}_{L_{K}}^{-1}(0) \cap Q^{-1}(1)$, which by hypothesis is not empty, $T_{v}\left(U_{\nu_{\mathrm{o}}}\right)_{K}=T_{v} \mathbf{J}_{L_{K}}^{-1}(0)+T_{v} Q^{-1}(1)$. Since $T_{v} Q^{-1}(1)$ has codimension one, it suffices to find a vector $w \in T_{v} \mathbf{J}_{L_{K}}^{-1}(0)$ that does not lie in $T_{v} Q^{-1}(1)$. This job is done by $w=\left.\frac{d}{d t}\right|_{t=0} v+t v \in T_{v}\left(U_{\nu_{\circ}}\right)_{K}$ since a straightforward calculation shows that, in one hand, $T_{v} \mathbf{J}_{L_{K}} \cdot w=0$ because $v \in \mathbf{J}_{L_{K}}^{-1}(0)$ and, at the same time, $\mathbf{d} Q(v) \cdot w=\mathbf{d}^{2} h(0)(v, v)=2 Q^{K}(v) \neq 0$, by the definiteness hypotheses on $Q^{K}$, hence $w \notin T_{v} Q^{-1}(1)$.

(ii) Since $\mathbf{J}_{L_{K}}^{-1}(\lambda) \cap Q^{-1}(1)=\mathbf{J}_{L_{K}}^{-1}(\lambda) \cap Q_{K}^{-1}(1) \subset\left(U_{\nu_{\circ}}\right)_{K}$ is a smooth submanifold of $U_{\nu_{\circ}}$ and $\left(U_{\nu_{\circ}}\right)_{K}$ is a smooth submanifold of $U_{\nu_{\circ}}$, then $\mathbf{J}_{L_{K}}^{-1}(\lambda) \cap Q^{-1}(1)$ is a smooth submanifold of $\left(U_{\nu_{\circ}}\right)_{K}$. We show the compactness of $\mathbf{J}_{L_{K}}^{-1}(\lambda) \cap Q^{-1}(1)$ by proving that it is sequentially compact, that is, any sequence $\left\{x_{n}\right\} \subset \mathbf{J}_{L_{K}}^{-1}(\lambda) \cap Q^{-1}(1)$ has a convergent subsequence in $\mathbf{J}_{L_{K}}^{-1}(\lambda) \cap Q^{-1}(1)$. Indeed, since $Q^{-1}(1)$ is a compact subset of $U_{\nu_{0}}$, there exists a subsequence $\left\{x_{n_{k}}\right\}$ of $\left\{x_{n}\right\}$, convergent in $Q^{-1}(1)$, that is $x_{n_{k}} \rightarrow l$, with $l \in Q^{-1}(1)$. Since $\left\{x_{n_{k}}\right\} \subset \mathbf{J}_{L_{K}}^{-1}(\lambda)$ for all $n_{k}$ and $\mathbf{J}_{L_{K}}^{-1}(\lambda)$ is closed then $l \in \mathbf{J}_{L_{K}}^{-1}(\lambda)$ necessarily, as required. The claim on the dimension is a consequence of the Transversality Theorem.

(iii) We proceed by contradiction: suppose that the point $v \in\left(U_{\nu_{\circ}}\right)_{K}$ is a relative equilibrium of the system with velocity $\xi \in \mathfrak{l}$. This implies that $\mathbf{d}\left(Q_{K}-\mathbf{J}_{L_{K}}^{\xi}\right)(v)=0$. The Lagrange Multipliers 
Theorem (taking $\xi$ in Proposition [2.6 as the Lagrange multiplier) implies that $\left.\mathbf{d} Q_{K}(v)\right|_{\operatorname{ker} T_{v} \mathbf{J}_{L_{K}}}=0$. However, the transversality $\mathbf{J}_{L_{K}}^{-1}(\lambda) \pitchfork Q_{K}^{-1}(1)$ implies the existence of vectors $w \in \operatorname{ker} T_{v} \mathbf{J}_{L_{K}}$ that do not belong to $T_{v} Q_{K}^{-1}(1)$ and therefore $\mathbf{d} Q_{K}(v) \cdot w \neq 0$, which represents a contradiction.

\subsubsection{Normal form formulation}

As we already said, the main idea behind the theorem consists of using the normal form theorem and Proposition 2.1 to reduce the search for RPOs with isotropy subgroup $K$ to the search for periodic orbits of the Hamiltonian systems of the form $\left(\left.\omega\right|_{V_{K}}, h_{\xi}\right)$ with $h_{\xi}:=\left.h\right|_{V_{K}}-\mathbf{J}_{L_{K}}^{\xi}$. We will first apply the ideas introduced in Section 2.2 to construct a normal form for these systems that will set up the problem of this search for periodic orbits in terms of the search for relative equilibria of a $S^{1}$-action that we will describe in what follows.

Firstly, consider the symmetric Hamiltonian system $\left(V^{K},\left.\omega\right|_{V^{K}},\left.h\right|_{V^{K}}, N(K) / K, \mathbf{J}_{L^{K}}: V^{K} \rightarrow \mathfrak{l}^{*}\right)$. The RPOs of this system amount to RPOs of the original system whose isotropy subgroups include $K$. Let $A^{K}:=D X_{\left.h\right|_{V} K}(0)$ be the linearization at zero of the Hamiltonian vector field $X_{\left.h\right|_{V} K}$. By the hypotheses on $X_{h}$, the eigenvalues $\pm i \nu_{\circ}$ are in the spectrum of $A^{K}$, and the corresponding resonance space is $U_{\nu_{0}}^{K}$. The mapping $\left.\theta \in S^{1} \mapsto \mathrm{e}^{\frac{\theta}{\nu_{0}} A^{K}}\right|_{U_{\nu_{0}}^{K}}$ generates a symplectic linear $S^{1}$-action on $\left(U_{\nu_{0}}^{K},\left.\omega\right|_{U_{\nu_{0}}^{K}}\right)$, whose momentum map is given by $\frac{1}{\nu_{0}} Q^{K}$.

Consider now $h_{\xi}^{K}:=\left.h\right|_{U_{\nu_{0}}^{K}}-\mathbf{J}_{L^{K}}^{\xi}$, with $\xi \in \mathfrak{l}$, as a $\mathfrak{l}$-parameter family of Hamiltonian functions on $U_{\nu_{\circ}}^{K}$ in the sense of point (v) in Section 2.2 Since for any $\xi \in \mathfrak{l}$ this family satisfies that $h_{\xi}^{K}(0)=0$, $\mathbf{d} h_{\xi}^{K}(0)=0$, and $D X_{h_{0}^{K}}(0)=D X_{\left.h\right|_{U_{\nu_{0}}^{K}}}(0)=\left.A\right|_{U_{\nu_{0}}^{K}}=A^{K}$ is non degenerate, we can construct a normal form equivalent system $\widehat{h_{\xi}^{K}}$ whose $S^{1}$-relative equilibria give us the periodic orbits of $h_{\xi}^{K}$. Due to the fact that the $N(K) / K$ and $S^{1}$-actions on $U_{\nu_{\circ}}^{K}$ commute and that $\mathbf{J}_{L^{K}}$ is quadratic, the normal form $\widehat{h_{\xi}^{K}}$ can be chosen so that

$$
\widehat{h_{\xi}^{K}}=\widehat{\left.h\right|_{\nu_{\nu^{K}}^{K}}}-\mathbf{J}_{L^{K}}^{\xi},
$$

with $\widehat{\left.h\right|_{\nu_{\nu_{\circ}}^{K}}}$ an $N(K) / K \times S^{1}$-invariant function on $U_{\nu_{\circ}}^{K}$ such that

$$
\widehat{\left.h\right|_{\nu_{\nu_{\mathrm{o}}}^{K}}}(u)=Q^{K}(u)+\left.\frac{1}{2} \mathbf{d}^{2} h\right|_{U_{\nu_{\mathrm{o}}}^{K}}(0)(u, u)+\cdots+\left.\frac{1}{k !} \mathbf{d}^{k} h\right|_{U_{\nu_{\mathrm{o}}}^{K}}(0)\left(u^{(k)}\right)+\text { (higher order terms). }
$$

\subsubsection{The critical points of the normal form Hamiltonian and a blow up argument}

In the following lemma we evaluate the critical points of the restriction of the function $\widehat{\left.h\right|_{U_{\nu_{0}}^{K}}}$ to the level sets of the form $\mathbf{J}_{L_{K}}^{-1}(\lambda) \cap Q_{K}^{-1}(\epsilon)$, where $\lambda \in B(K)$, the neighborhood of zero in $\mathfrak{l}^{*}$ introduced in Proposition 3.8 and $\epsilon>0$ is very small. Furthermore, a blow up argument will show that these critical points can be arranged in smooth branches.

Lemma 3.9 Suppose that we are under the hypotheses of Theorem 3.1. Then, the restriction of the function $h_{k} \in C^{\infty}\left(U_{\nu_{0}}^{K}\right)$ defined by $h_{k}(u):=\left.\frac{1}{k !} \mathbf{d}^{k} h\right|_{U_{\nu_{0}}^{K}}(0)\left(u^{(k)}\right)$, to the level sets of the form $\mathbf{J}_{L_{K}}^{-1}(\lambda) \cap$ $Q_{K}^{-1}(1)$, where $\lambda \in B(K)$, the neighborhood of zero in $\mathfrak{l}^{*}$ introduced in Proposition 3.8 , has at least

$$
\max \left[\operatorname{Cat}\left(\mathbf{J}_{L_{K}}^{-1}(\lambda) \cap Q_{K}^{-1}(1) /\left(L_{\lambda} \times S^{1}\right)\right), \chi\left(\mathbf{J}_{L_{K}}^{-1}(\lambda) \cap Q_{K}^{-1}(1)\right)^{L_{\lambda} \times S^{1}}\right]
$$

distinct critical points.

Furthermore, let $\lambda_{\circ} \in B(K) \subset \mathfrak{l}^{*}$ be arbitrary but fixed and let $\left\{u_{1}, \ldots, u_{k}\right\}$ be the set of critical points of the restriction of the function $h_{k}$ to the level set $\mathbf{J}_{L_{K}}^{-1}\left(\lambda_{\circ}\right) \cap Q_{K}^{-1}(1)$, provided by 3.4). Then, for each $u_{i}, i \in\{1, \ldots, k\}$, there exist a neighborhood $E \subset \mathbb{R}$ of the origin in the real line, a neighborhood $V_{\lambda_{\circ}} \subset \mathfrak{l}^{*}$ of $\lambda_{\circ}$ in $\mathfrak{l}^{*}$, and a smooth function $\rho: E \times V_{\lambda_{\circ}} \rightarrow Q_{K}^{-1}(1)$ such that $\rho\left(0, \lambda_{\circ}\right)=u_{i}$ and also, the function $v: E \times V_{\lambda_{\circ}} \rightarrow U_{\nu_{\circ}}^{K}$ defined by $v(r, \lambda):=r \rho(r, \lambda)$ satisfies that: 
(i) $v(r, \lambda) \in\left(U_{\nu_{\mathrm{o}}}\right)_{K}$ iff $r \neq 0$.

(ii) $Q^{K}(v(r, \lambda))=r^{2}$ and $\mathbf{J}_{L^{K}}(v(r, \lambda))=r^{2} \lambda, r \in E, \lambda \in V_{\lambda_{\circ}}$.

(iii) $\left.\mathbf{d} \widehat{\left.h\right|_{U_{\mathrm{o}}^{K}}}\right|_{\mathbf{J}_{L^{K}}^{-1}\left(r^{2} \lambda\right) \cap\left(Q^{K}\right)^{-1}\left(r^{2}\right)}(v(r, \lambda))=0$, that is, the branches $v(r, \lambda)$ are made of critical points of the restriction of the function $\widehat{\left.h\right|_{U_{\nu_{0}}^{K}}}$ to the level sets $\mathbf{J}_{L^{K}}^{-1}\left(r^{2} \lambda\right) \cap\left(Q^{K}\right)^{-1}\left(r^{2}\right)$.

Proof Firstly, note that the estimate (3.4) is a straightforward consequence of Proposition [2.2 (2.7), and the invariance properties of $h_{k}$.

We now prove the existence of the branches in the statement. Let $u_{0}$ be one of the critical points of the restriction of the function $h_{k}$ to the level set $\mathbf{J}_{L_{K}}^{-1}\left(\lambda_{\circ}\right) \cap Q_{K}^{-1}(1)$, provided by (3.4). The transversality of $\mathbf{J}_{L_{K}}$ and $Q_{K}$ implies the existence of a very convenient coordinate patch around $u_{0}$ in $Q_{K}^{-1}(1)$. In order to construct it, let us show first that the restriction $\left.\mathbf{J}_{L_{K}}\right|_{Q_{K}^{-1}(1) \cap \mathbf{J}_{L_{K}}^{-1}(B(K))}$ is a submersion onto its image. Indeed, for any $u \in Q_{K}^{-1}(1) \cap \mathbf{J}_{L_{K}}^{-1}(B(K))$ we have that

$$
\begin{aligned}
\operatorname{dim} \operatorname{Im} & \left(\left.T_{u} \mathbf{J}_{L_{K}}\right|_{Q_{K}^{-1}(1) \cap \mathbf{J}_{L_{K}}^{-1}(B(K))}\right)=\operatorname{dim}\left(\operatorname{ker} T_{u} Q_{K}\right)-\operatorname{dim}\left(\left.\operatorname{ker} T_{u} \mathbf{J}_{L_{K}}\right|_{Q_{K}^{-1}(1)}\right) \\
& =\operatorname{dim} U_{\nu_{\circ}}^{K}-1-\operatorname{dim}\left(\operatorname{ker} T_{u} \mathbf{J}_{L_{K}} \cap \operatorname{ker} T_{u} Q_{K}\right) \\
& =\operatorname{dim} U_{\nu_{\circ}}^{K}-1+\operatorname{dim}\left(\operatorname{ker} T_{u} \mathbf{J}_{L_{K}}+\operatorname{ker} T_{u} Q_{K}\right)-\operatorname{dim}\left(\operatorname{ker} T_{u} \mathbf{J}_{L_{K}}\right)-\operatorname{dim}\left(\operatorname{ker} T_{u} Q_{K}\right) \\
& =\operatorname{dim} \mathfrak{l}^{*},
\end{aligned}
$$

as required. In the last equality we used that since $u \in \mathbf{J}_{L_{K}}^{-1}(B(K))$, then $\operatorname{ker} T_{u} \mathbf{J}_{L_{K}} \pitchfork \operatorname{ker} T_{u} Q_{K}$, and therefore $\operatorname{dim}\left(\operatorname{ker} T_{u} \mathbf{J}_{L_{K}}+\operatorname{ker} T_{u} Q_{K}\right)=\operatorname{dim} U_{\nu_{0}}^{K}$. In these circumstances, the Local Submersion Theorem (see for instance [2, Theorem 3.5.2]) implies the existence of a neighborhood $V_{\lambda_{\circ}}$ of $\lambda_{\circ}$ in $\mathfrak{l}^{*}$, a neighborhood $W$ of the origin in $\mathbb{R}^{s}$, with $s=\operatorname{dim} U_{\nu_{\circ}}^{K}-\operatorname{dim} \mathfrak{l}^{*}-1$, and a mapping $\varphi: V_{\lambda_{\circ}} \times W \rightarrow Q_{K}^{-1}(1)$ that is a diffeomorphism onto its image, such that:

$$
\varphi\left(\lambda_{\circ}, 0\right)=u_{0} \quad \text { and } \quad \mathbf{J}_{L_{K}}(\varphi(\lambda, w))=\lambda, \quad \lambda \in V_{\lambda_{\circ}}, w \in W .
$$

We will further improve this coordinate patch around $u_{0}$ by "factoring out" the $L_{\lambda_{\circ}} \times S^{1}$-action in it. Indeed, since the Lie group $L_{\lambda_{\circ}} \times S^{1}$ acts on $Q_{K}^{-1}(1)$, we can induce a local action of this group on $V_{\lambda_{\circ}} \times W$ by declaring $\varphi$ to be equivariant, that is, for any $g=(l, \theta) \in L_{\lambda_{\circ}} \times S^{1}$ close enough to the identity we define $g \cdot(\lambda, w):=\varphi^{-1}(g \cdot \varphi(\lambda, w))$. Note that by the very definition of this action and by (3.5), we have that

$$
\mathbf{J}_{L_{K}}(\varphi(g \cdot(\lambda, w)))=\mathbf{J}_{L_{K}}\left(\varphi\left(\varphi^{-1}(g \cdot \varphi((\lambda, w)))\right)\right)=\mathbf{J}_{L_{K}}(g \cdot \varphi(\lambda, w))=l \cdot \mathbf{J}_{L_{K}}(\varphi(\lambda, w))=l \cdot \lambda,
$$

consequently

$$
g \cdot(\lambda, w)=(l, \theta) \cdot(\lambda, w)=(l \cdot \lambda, \Phi(g, \lambda, w)),
$$

for some smooth function $\Phi$. The dot in $l \cdot \lambda$ denotes the coadjoint action of $L_{\lambda_{\circ}}$ on $\mathfrak{r}^{*}$. Note that in (3.6) we used the equivariance of $\mathbf{J}_{L_{K}}$ with respect to the $L_{\lambda_{\circ}}$-action that was inherited from its $L$-equivariance, as well as the invariance of $\mathbf{J}_{L_{K}}$ with respect to the $S^{1}$-action which is justified, via Noether's Theorem, by the fact that this action is induced by a Hamiltonian flow associated to a $L$-invariant Hamiltonian, namely $Q^{K}$.

Using these remarks we are now going to construct a slice for the local $L_{\lambda_{\diamond}} \times S^{1}$-action on $V_{\lambda_{\circ}} \times W$ that goes through the point $\left(\lambda_{\circ}, 0\right)$. Firstly, it is easy to see by using (3.7) that

$$
T_{\left(\lambda_{\circ}, 0\right)}\left(\left(L_{\lambda_{\circ}} \times S^{1}\right) \cdot\left(\lambda_{\circ}, 0\right)\right)=\{0\} \times W^{\prime},
$$

where $W^{\prime} \subset W$ is a vector subspace of $W$. The remarks that we made in Section [2.1 about the construction of the slices implies the existence of a smooth mapping $\psi$ diffeomorphic onto its image of the form

$$
\begin{aligned}
& \psi: V_{\lambda_{\circ}} \times U \quad \longrightarrow \quad V_{\lambda_{\circ}} \times W \\
& (\lambda, u) \longmapsto(\lambda, \eta(\lambda, u))
\end{aligned}
$$


whose image (shrinking $V_{\lambda_{\circ}}$ if necessary) is a local slice through $\left(\lambda_{\circ}, 0\right)$ for the local $L_{\lambda_{\circ}} \times S^{1}$-action on $V_{\lambda_{0}} \times W$. The set $U$ is an open neighborhood of the origin in a vector space isomorphic to $W / W^{\prime}$ and $\eta: V_{\lambda_{\circ}} \times U \rightarrow V_{\lambda_{\circ}} \times W$ is a smooth map such that $\eta\left(\lambda_{\circ}, 0\right)=0$.

In these circumstances, the version of the Slice Theorem that we presented in Section 2.1 implies the existence of a local cross-section $\sigma: \mathcal{Z} \subset L_{\lambda_{\circ}} \times S^{1} /\left(L_{\lambda_{\circ}} \times S^{1}\right)_{u_{0}} \rightarrow L_{\lambda_{\circ}} \times S^{1}$ of the homogeneous space $L_{\lambda_{\circ}} \times S^{1} /\left(L_{\lambda_{\diamond}} \times S^{1}\right)_{u_{0}}$, and a smooth map $F$ of the form

$$
\begin{array}{ccc}
F: \mathcal{Z} \times V_{\lambda_{\circ}} \times U & \longrightarrow & Q_{K}^{-1}(1) \\
(z, \lambda, u) & \longmapsto & \sigma(z) \cdot \varphi(\lambda, \eta(\lambda, u))
\end{array}
$$

that is a diffeomorphism onto an open set of $Q_{K}^{-1}(1)$ that contains $u_{0}$.

We will now use this coordinate patch to obtain the branches $v(r, \lambda)$ whose existence we claim in the second part of the statement. We start by setting up the problem in polar coordinates since it is a polar blowing-up argument what will give us the result. Let $2 n$ be the dimension of the symplectic vector space $U_{\nu_{\circ}}^{K}$. We will denote by $S^{2 n-1}$ the sphere in $U_{\nu_{\circ}}^{K}$ obtained by using the norm associated to the definite quadratic form $Q^{K}$. We now define the blown-up Hamiltonian $h^{b}: \mathbb{R} \times S^{2 n-1} \rightarrow \mathbb{R}$, as:

$$
h^{b}(r, u):=\widehat{\left.h\right|_{U_{\nu_{0}}^{K}}}(r u), \quad r \in \mathbb{R}, \quad u \in S^{2 n-1} .
$$

When the variable $u$ is in a neighborhood of $u_{0}$, we can use (3.8) to give a local expression for $h^{b}$ in $\mathcal{Z} \times V_{\lambda_{\circ}} \times U$-variables; let $h^{l b}: \mathbb{R} \times \mathcal{Z} \times V_{\lambda_{\circ}} \times U \rightarrow \mathbb{R}$ be the local expression of the blown-up Hamiltonian, defined by

$$
h^{l b}(r, z, \lambda, u):=h^{b}(r(\sigma(z) \cdot \varphi(\lambda, \eta(\lambda, u))))=\widehat{\left.h\right|_{\nu_{\mathrm{o}}^{K}} ^{K}}(r(\sigma(z) \cdot \varphi(\lambda, \eta(\lambda, u)))) .
$$

Notice that the $G$-invariance of the Hamiltonian $h$ implies that

$$
h^{l b}(r, z, \lambda, u)=\widehat{\left.h\right|_{U_{\nu_{\mathrm{o}}}^{K}}}(r(\sigma(z) \cdot \varphi(\lambda, \eta(\lambda, u))))=\widehat{\left.h\right|_{U_{\nu_{\mathrm{o}}}^{K}}}(r \varphi(\lambda, \eta(\lambda, u))) \equiv h^{l b}(r, \lambda, u),
$$

that is, $h^{l b}$ does not depend on the $\mathcal{Z}$-variables. The main advantage of the use of these coordinates is the fact we can search for the critical points of the restriction of $\widehat{\left.h\right|_{\nu_{\nu}^{K}} ^{K}}$ to the level sets $\mathbf{J}_{L^{K}}^{-1}(\lambda) \cap\left(Q^{K}\right)^{-1}(\epsilon)$ by looking for the triples $(r, \lambda, u) \in \mathbb{R} \times V_{\lambda_{\circ}} \times U$ for which $D_{U} h^{l b}(r, \lambda, u)=0\left(D_{U} h^{l b}\right.$ denotes the partial Fréchet derivative of $h^{l b}$ relative to the $U$-variable). More specifically: $D_{U} h^{l b}(r, \lambda, u)=0$ iff the restriction of $\widehat{\left.h\right|_{U_{\nu}^{K}} ^{K}}$ to the level set $\mathbf{J}_{L^{K}}^{-1}\left(r^{2} \lambda\right) \cap\left(Q^{K}\right)^{-1}(r)$ has a critical point at $r \varphi(\lambda, \eta(\lambda, u))$. Using now hypotheses (H1) and (H2) on the Hamiltonian $h$ we can write

$$
h^{l b}(r, \lambda, u)=r^{2}+\alpha_{4} r^{4}+\alpha_{6} r^{6}+\cdots+\alpha_{k-2} r^{k-2}+r^{k} h_{k}^{l b}(\lambda, u)+o\left(r^{k}, \lambda, u\right),
$$

where $\alpha_{4}, \alpha_{6}, \ldots, \alpha_{k-2}$ are real coefficients and $h_{k}^{l b}(\lambda, u):=h_{k}(\varphi(\lambda, \eta(\lambda, u)))$. Expression (3.9) can be rewritten of the form

$$
h^{l b}(r, \lambda, u)-r^{2}-\alpha_{4} r^{4}-\alpha_{6} r^{6}-\cdots-\alpha_{k-2} r^{k-2}=r^{k} g(r, \lambda, u),
$$

with $g$ is a smooth function on his variables such that

$$
D_{U} g(r, \lambda, u)=0 \quad \text { if and only if } \quad D_{U} h^{l b}(r, \lambda, u)=0 .
$$

The Taylor expansion of $g$ on the $r$ variables around $r=0$ has the form

$$
g(r, \lambda, u)=h_{k}^{l b}(\lambda, u)+o(r, \lambda, u)=h_{k}(\varphi(\lambda, \eta(\lambda, u)))+o(r, \lambda, u)
$$

Notice that

$$
g\left(0, \lambda_{\circ}, 0\right)=h_{k}\left(\varphi\left(\lambda_{\circ}, \eta\left(\lambda_{\circ}, 0\right)\right)\right)=h_{k}\left(u_{0}\right)
$$

and that

$$
D_{U} g\left(0, \lambda_{\circ}, 0\right)=D_{U} h_{k}^{l b}\left(\lambda_{\circ}, 0\right)=\mathbf{d} h_{k}\left(u_{0}\right) \cdot\left(D_{W} \varphi\left(\lambda_{\circ}, 0\right) \cdot D_{U} \eta\left(\lambda_{\circ}, 0\right)\right)=0
$$


since $D_{W} \varphi\left(\lambda_{\circ}, 0\right) \cdot D_{U} \eta\left(\lambda_{\circ}, 0\right)$ maps into $T_{u_{0}}\left(\mathbf{J}_{L_{K}}^{-1}\left(\lambda_{\circ}\right) \cap Q_{K}^{-1}(1)\right)$, which, by the choice of $u_{0}$ lies in the kernel of $\mathbf{d} h_{k}\left(u_{0}\right)$ (recall that $u_{0}$ was chosen to be a critical point of the restriction of $h_{k}$ to $\left.\mathbf{J}_{L_{K}}^{-1}\left(\lambda_{\circ}\right) \cap Q_{K}^{-1}(1)\right)$. Moreover, it is easy to see that for any pair $u, v \in U$ :

$D_{U}^{2} g\left(0, \lambda_{\circ}, 0\right)(u, v)=\left.\mathbf{d}^{2} h_{k}\right|_{\mathbf{J}_{L_{K}}^{-1}\left(\lambda_{\circ}\right) \cap Q_{K}^{-1}(1)}\left(u_{0}\right)\left(D_{W} \varphi\left(\lambda_{\circ}, 0\right) \cdot D_{U} \eta\left(\lambda_{\circ}, 0\right) \cdot u, D_{W} \varphi\left(\lambda_{\circ}, 0\right) \cdot D_{U} \eta\left(\lambda_{\circ}, 0\right) \cdot v\right)$.

The $(N(K) / K)_{\lambda} \times S^{1}$-Morse condition on $\left.h_{k}\right|_{\mathbf{J}_{L_{K}}^{-1}\left(\lambda_{\circ}\right) \cap Q_{K}^{-1}(1)}$ implies that $D_{U}^{2} g\left(0, \lambda_{\circ}, 0\right)$ is a non degenerate quadratic form since the image of the linear mapping $D_{W} \varphi\left(\lambda_{\circ}, 0\right) \cdot D_{U} \eta\left(\lambda_{\circ}, 0\right): U \rightarrow T_{u_{0}}\left(\mathbf{J}_{L_{K}}^{-1}\left(\lambda_{\circ}\right) \cap\right.$ $\left.Q_{K}^{-1}(1)\right)$ is a vector subspace of $T_{u_{0}}\left(\mathbf{J}_{L_{K}}^{-1}\left(\lambda_{\circ}\right) \cap Q_{K}^{-1}(1)\right)$ that, by construction, is complementary to $T_{u_{0}}\left(\left(L_{\lambda_{\circ}} \times S^{1}\right) \cdot u_{0}\right)$. In this situation the Implicit Function Theorem guarantees the existence of a function $u: E \times V_{\lambda_{\circ}} \rightarrow U$ (shrink $V_{\lambda_{\circ}}$ if necessary) with $E \subset \mathbb{R}$ a neighborhood of the origin in $\mathbb{R}$, such that

$$
D_{U} g(r, \lambda, u(r, \lambda))=0
$$

which, by (3.10) is equivalent to having $D_{U} h^{l b}(r, \lambda, u(r, \lambda))=0$ and consequently

$$
\left.\mathbf{d} \widehat{h_{U_{\nu_{\mathrm{o}}^{K}}}}\right|_{\mathbf{J}_{L^{K}}^{-1}\left(r^{2} \lambda\right) \cap\left(Q^{K}\right)^{-1}(r)}(r \varphi(\lambda, \eta(\lambda, u(r, \lambda))))=0 .
$$

The claim of the Lemma follows by taking $\rho(r, \lambda):=\varphi(\lambda, \eta(\lambda, u(r, \lambda)))$ and $v(r, \lambda):=r \rho(r, \lambda)=$ $r \varphi(\lambda, \eta(\lambda, u(r, \lambda)))$.

\subsubsection{Lagrange multipliers and RPOs}

We now show that the branches of critical points found in Lemma 3.9 amount to RPOs of the original system.

Let now $\lambda_{\circ} \in \mathfrak{l}$ and $v\left(r, \lambda\right.$ ) (with $v: E \times V_{\lambda_{\circ}} \rightarrow U_{\nu_{\circ}}^{K}$ ) be one of the branches of critical points of the restriction of the function $\widehat{h_{U_{\nu_{0}}^{K}}}$ to the level sets $\mathbf{J}_{L^{K}}^{-1}\left(r^{2} \lambda\right) \cap\left(Q^{K}\right)^{-1}\left(r^{2}\right)$ provided by (3.4). Proposition 2.6 guarantees, for each $v(r, \lambda)$ the existence of a multiplier $(\Lambda(r, \lambda), c(r, \lambda)) \in \mathfrak{l} \times \mathbb{R}$ such that:

$$
\mathbf{d} \widehat{\left.h\right|_{U_{\nu_{\mathrm{o}}}^{K}}}(v(r, \lambda))=c(r, \lambda) \mathbf{d} Q^{K}(v(r, \lambda))+\mathbf{d} \mathbf{J}_{L^{K}}^{\Lambda(r, \lambda)}(v(r, \lambda)),
$$

which implies that the point $v(r, \lambda)$ is a periodic point of $X_{h_{\Lambda(r, \lambda)}^{K}}$. If we are able to show that $\Lambda(r, \lambda)$ can be made very small so that we can use the Normal Form Theorem, all these periodic points will amount to periodic orbits of $X_{h_{U_{\nu_{0}}^{K}}-\mathbf{J}_{L K}^{\Lambda(r, \lambda)}}$ and, by Proposition 2.1] to RPOs of $X_{h}$. We will prove this point by pairing both sides of (3.11) at the point $v\left(r, \lambda_{\circ}\right)$ with the vector $w_{\lambda}:=D_{\lambda} v\left(r, \lambda_{\circ}\right) \cdot \lambda, \lambda \in \mathfrak{l}^{*}$ arbitrary, and taking into account that by the very construction of the function $v(r, \lambda), \mathbf{J}_{L^{K}}(v(r, \lambda))=r^{2} \lambda$, for any $r \in E$ and any $\lambda \in V_{\lambda_{\circ}}$. Indeed,

$$
\mathbf{d} \widehat{\left.h\right|_{U_{\nu_{\circ}}^{K}}}\left(v\left(r, \lambda_{\circ}\right)\right) \cdot w_{\lambda}=c\left(r, \lambda_{\circ}\right) \mathbf{d} Q^{K}\left(v\left(r, \lambda_{\circ}\right)\right) \cdot w_{\lambda}+\mathbf{d} \mathbf{J}_{L^{K}}^{\Lambda\left(r, \lambda_{\circ}\right)}\left(v\left(r, \lambda_{\circ}\right)\right) \cdot w_{\lambda} .
$$

Note first that $\mathbf{d} Q^{K}\left(v\left(r, \lambda_{\circ}\right)\right) \cdot w_{\lambda}=0$ for all $\lambda \in \mathfrak{l}^{*}$. Also,

$$
\begin{aligned}
\mathbf{d} \mathbf{J}_{L^{K}}^{\Lambda\left(r, \lambda_{\circ}\right)}\left(v\left(r, \lambda_{\circ}\right)\right) \cdot w_{\lambda}=\left.\frac{d}{d t}\right|_{t=0} \mathbf{J}_{L^{K}}^{\Lambda\left(r, \lambda_{\circ}\right)}\left(v\left(r, \lambda_{\circ}+t \lambda\right)\right) & \\
& =\left.\frac{d}{d t}\right|_{t=0}\left\langle r^{2}\left(\lambda_{\circ}+t \lambda\right), \Lambda\left(r, \lambda_{\circ}\right)\right\rangle=r^{2}\left\langle\lambda, \Lambda\left(r, \lambda_{\circ}\right)\right\rangle .
\end{aligned}
$$

This equality implies, together with hypotheses (H1) and (H2) that the multiplier $\Lambda(r, \lambda)$ is a smooth function in its variables $\Lambda: E \times V_{\lambda_{\circ}} \rightarrow \mathfrak{l}$ since it can be written as a composition of smooth functions, namely

$$
\Lambda(r, \lambda)=\frac{1}{r^{2}} \mathbf{d} \widehat{\left.h\right|_{U_{\nu^{\prime}}^{K}}}(v(r, \lambda)) \cdot D_{\lambda} v(r, \lambda) .
$$


Even though in the previous equality it seems that there is a singularity at $r=0$ we see in what follows that it is not the case. Indeed, by hypothesis (H2) we can write

$$
\widehat{\left.h\right|_{U_{\nu_{\mathrm{o}}}^{K}}}(v(r, \lambda))=r^{2}+\alpha_{4} r^{4}+\alpha_{6} r^{6}+\cdots+\alpha_{k-2} r^{k-2}+r^{k} h_{k}(\rho(r, \lambda))+o\left(r^{k}, \lambda\right),
$$

where $\alpha_{4}, \alpha_{6}, \ldots, \alpha_{k-2}$ are real coefficients. It is easy to see from (3.15) that for any $\eta \in \mathfrak{l}^{*}$

$$
\mathbf{d} \widehat{\left.h\right|_{U_{\nu_{0}}^{K}}}(v(r, \lambda)) \cdot w_{\eta}=r^{k} \mathbf{d} h_{k}(\rho(r, \lambda)) \cdot D_{\lambda} \rho(r, \lambda) \cdot \eta+o\left(r^{k}, \lambda\right) .
$$

Given that by hypothesis (H2) $k \geq 4$, expression (3.14) can be rewritten as

$$
\Lambda(r, \lambda)=r^{k-2} \mathbf{d} h_{k}(\rho(r, \lambda)) \cdot D_{\lambda} \rho(r, \lambda) \cdot \eta+o\left(r^{k-2}, \lambda\right),
$$

where the smoothness of the function $\Lambda(r, \lambda)$ is apparent as well as the fact that $\Lambda(0, \lambda)=0$. These two points together imply that the multiplier $\Lambda(r, \lambda)$ can be made as small as we want by taking $r$ sufficiently close to the origin, as desired. This allows us to use the Normal Form Theorem to conclude that for $r$ small enough, the RPOs of $\widehat{\left.h\right|_{U_{\nu^{K}}^{K}}}$ amount to RPOs of the original system.

We will now prove that the RPOs that we just obtained have relative periods close to $T_{\nu_{\circ}}$ by showing that as $r$ tends to zero, the multiplier $c(r, \lambda)$ approaches to 1 . We prove this point by pairing both sides of (3.11) with the vector $u:=D_{r} v(r, \lambda) \cdot 1$. First of all

$$
\mathbf{d} Q^{K}(v(r, \lambda)) \cdot u=\left.\frac{d}{d t}\right|_{t=0} Q^{K}(v(r+t, \lambda))=\left.\frac{d}{d t}\right|_{t=0}(r+t)^{2}=2 r .
$$

Also,

$$
\begin{aligned}
\mathbf{d J}_{L^{K}}^{\Lambda(r, \lambda)}(v(r, \lambda)) \cdot u=\left.\frac{d}{d t}\right|_{t=0}\left\langle\mathbf{J}_{L^{K}}(v(r+t, \lambda)),\right. & \Lambda(r, \lambda)\rangle \\
& =\left.\frac{d}{d t}\right|_{t=0}\left\langle(r+t)^{2} \lambda, \Lambda(r, \lambda)\right\rangle=2 r\langle\lambda, \Lambda(r, \lambda)\rangle .
\end{aligned}
$$

From expression (3.15) it is easy to see that $\mathbf{d} \widehat{h_{U_{\nu_{0}}^{H}}}(v(r, \lambda)) \cdot u=2 r+o(r, \lambda)$. This equality, together with (3.16) and (3.17) imply, when substituted in (3.11) paired with $u$ that

$$
1-c(r, \lambda)=\langle\lambda, \Lambda(r, \lambda)\rangle+o(r, \lambda) .
$$

Since $\Lambda(r, \lambda) \rightarrow 0$ when $r$ tends to zero then $c(r, \lambda) \rightarrow 1$ as $r \rightarrow 0$, as claimed.

\subsubsection{Dimensional estimate of the Lusternik-Schnirelmann category}

In order to conclude the proof of the theorem we will give a dimensional estimate of the first part of the estimate on the branches (3.4) whose relatively periodic character we just proved. In order to obtain the claim (3.1) in the statement of the theorem we just need to show that

$$
\operatorname{Cat}\left(\mathbf{J}_{L_{K}}^{-1}(\lambda) \cap Q_{K}^{-1}(1) /\left(L_{\lambda} \times S^{1}\right)\right) \geq \frac{1}{2}\left[\operatorname{dim}\left(U_{\nu_{\circ}}\right)_{K}-\operatorname{dim}(N(K) / K)-\operatorname{dim}(N(K) / K)_{\lambda}\right] .
$$

We will prove this inequality with the help of Proposition 2.3 taking in its statement $\mathbf{J}_{L_{K}}^{-1}(\lambda) \cap Q_{K}^{-1}(1)$ as the submanifold $N$ and $L_{\lambda} \times S^{1}$ the subgroup $K$. Given that the mapping $\mathbf{J}_{L_{K}} \times \frac{1}{\nu_{\mathrm{o}}} Q_{K}$ is the momentum map corresponding to the $N(K) / K \times S^{1}$-action on $\left(U_{\nu_{\mathrm{o}}}\right)_{K}$, the Reduction Lemma (see for instance [1] Lemma 4.3.2]) guarantees the technical hypotheses (2.3), namely:

$$
\left(T_{z}\left(\mathbf{J}_{L_{K}}^{-1}(\lambda) \cap Q_{K}^{-1}(1)\right)\right)^{\omega}=\left(\operatorname{ker} T_{z}\left(\mathbf{J}_{L_{K}} \times \frac{1}{\nu_{\circ}} Q_{K}\right)\right)^{\omega}=T_{z}\left(\left(N(K) / K \times S^{1}\right) \cdot z\right), \quad \text { as well as, }
$$




$$
T_{z}\left(\mathbf{J}_{L_{K}}^{-1}(\lambda) \cap Q_{K}^{-1}(1)\right) \cap T_{z}\left(\left(N(K) / K \times S^{1}\right) \cdot z\right)=T_{z}\left(\left(L_{\lambda} \times S^{1}\right) \cdot z\right),
$$

for any $z \in \mathbf{J}_{L_{K}}^{-1}(\lambda) \cap Q_{K}^{-1}(1)$. In order to apply Proposition 2.3 we need the isotropy subgroups of any point in $\mathbf{J}_{L_{K}}^{-1}(\lambda) \cap Q_{K}^{-1}(1)$ to be a finite subgroup of $L_{\lambda} \times S^{1}$. This is so by the freeness and the local freeness of the $L_{\lambda}$ and $S^{1}$-actions on $\left(U_{\nu_{\mathrm{o}}}\right)_{K}$, respectively, and by the fact proved in the third part of Proposition 3.8 that $\mathbf{J}_{L_{K}}^{-1}(\lambda) \cap Q_{K}^{-1}(1)$ does not contain any $N(K) / K$-relative equilibria of the system $\left(\left(U_{\nu_{\mathrm{o}}}\right)_{K},\left.\omega\right|_{\left(U_{\nu_{0}}\right)_{K}},\left.Q\right|_{\left(U_{\nu_{0}}\right)_{K}}\right)$ that would be the only elements that could make the $L_{\lambda} \times S^{1}$-action not be locally free. Consequently, by Proposition 2.3 there is a cohomology class $\theta \in K^{2}\left(\mathbf{J}_{L_{K}}^{-1}(\lambda) \cap\right.$ $\left.Q_{K}^{-1}(1) /\left(L_{\lambda} \times S^{1}\right) ; \mathbb{R}\right)$ such that $\theta^{k} \neq 0$, with $k=\frac{1}{2}\left(\operatorname{dim}\left(\mathbf{J}_{L_{K}}^{-1}(\lambda) \cap Q_{K}^{-1}(1)\right)-\operatorname{dim}\left(L_{\lambda} \times S^{1}\right)\right)$. Therefore, the cuplength of $\mathbf{J}_{L_{K}}^{-1}(\lambda) \cap Q_{K}^{-1}(1) /\left(L_{\lambda} \times S^{1}\right)$ is at least

$$
\frac{1}{2}\left[\operatorname{dim}\left(\mathbf{J}_{L_{K}}^{-1}(\lambda) \cap Q_{K}^{-1}(1)\right)-\operatorname{dim} L_{\lambda}-1\right]=\frac{1}{2}\left[\operatorname{dim}\left(U_{\nu_{\circ}}\right)_{K}-\operatorname{dim} L-\operatorname{dim} L_{\lambda}-2\right],
$$

and hence, Proposition 2.4 establishes the inequality (3.18), which concludes the proof of the theorem.

Remark 3.10 The choice of the neighborhood $B(K)$ in Proposition 3.8 guarantees that the RPOs that we found in our theorem are nontrivial, that is, they are not just relative equilibria. Indeed, when $\lambda \in B(K)$, the level set of $Q_{K}^{-1}(1)$ intersects transversely the level set $\mathbf{J}_{L_{K}}^{-1}(\lambda)$, which in part (iii) of Proposition 3.8 allowed us to prove the inexistence of relative equilibria of the Hamiltonian vector field $X_{Q_{K}}$ with respect to the $N(K) / K$-symmetry in $\mathbf{J}_{L_{K}}^{-1}(\lambda) \cap Q_{K}^{-1}(1)$. Since the Hamiltonian $\left.h\right|_{\left(U_{\nu_{\circ}}\right)_{K}}$ can be considered as a perturbation of $Q_{K}$ for vectors of norm $r$ small enough, the transversality (which is a stable property) of $\left.h\right|_{\left(U_{\nu^{\circ}}\right)_{K}} ^{-1}(r)$ with respect to $\mathbf{J}_{L_{K}}^{-1}\left(r^{2} \lambda\right)$ is still valid, and therefore the inexistence of $N(H) / H$-relative equilibria in $\left.\mathbf{J}_{L_{K}}^{-1}\left(r^{2} \lambda\right) \cap h\right|_{\left(U_{\nu^{\circ}}\right)_{H}} ^{-1}(r)$ as well.

Remark 3.11 Even though the hypothesis (H1) appears in the proof of the theorem as a technical necessity, it turns out when it fails, the existence of genuine RPOs that are not either relative equilibria or plain periodic orbits is not possible. Indeed, suppose that the restriction $\left.h\right|_{U_{\nu_{0}}^{K}}$ is purely radial. In that case, $\left.h\right|_{U_{\nu_{0}}^{K}}$ is directly in normal form and there exists a real smooth function $f: \mathbb{R} \rightarrow \mathbb{R}$ such that $\left.h\right|_{U_{\nu_{0}}^{K}}(v)=f\left(Q^{K}(v)\right), v \in U_{\nu_{\circ}}^{K}$. In these circumstances, expression (3.11) reduces to

$$
f^{\prime}\left(Q^{K}(v(r, \lambda))\right) \mathbf{d} Q^{K}(v(r, \lambda))=c(r, \lambda) \mathbf{d} Q^{K}(v(r, \lambda))+\mathbf{d} \mathbf{J}_{L^{K}}^{\Lambda(r, \lambda)}(v(r, \lambda)),
$$

which amounts to

$$
\left.\mathbf{d} h\right|_{U_{\nu_{\mathrm{o}}}^{K}}(v(r, \lambda))-\mathbf{d} \mathbf{J}_{L^{K}}^{\frac{\Lambda(r, \lambda) f^{\prime}\left(Q^{K}(v(r, \lambda))\right)}{\left.f^{\prime}(v(r, \lambda))\right)-c(r, \lambda)}}(v(r, \lambda))=0,
$$

that is, in the absence of hypothesis $(\mathbf{H 1}) v(r, \lambda)$ is a branch of relative equilibria of the Hamiltonian vector field associated to $h$ (the reader interested in the technology for searching relative equilibria in the hypotheses of Theorem [3.1] or even weaker, can check with [11, 56], and references therein). Note that a trivial corollary of this comment is that if $h$ is just quadratic and therefore its associated Hamiltonian vector field is linear, then there are no genuine RPOs associated to its dynamics. On other words, the relative periodic orbits around stable equilibria are purely non linear phenomena.

\subsection{Relative periodic orbits with prescribed spatiotemporal symmetry}

In the statement of Theorem 3.1 we optimized the search for the RPOs of our system by looking for them within the fixed point spaces corresponding to the isotropy subspaces of the $G$-action on $U_{\nu_{\circ}}$. In Section 2.2 we showed that the resonance space $U_{\nu_{\circ}}$ can actually be endowed with a $G \times S^{1}$-symmetry which obviously contains more isotropy subgroups than merely the $G$-symmetry and that we could therefore utilize to obtain additional relatively periodic solutions. However, the reader should not forget that the $S^{1}$-symmetry is a feature owned solely by the system in normal form; the real system that 
we are dealing with is not $S^{1}$-symmetric. This fact does not pose a problem since the morphism that relates the $S^{1}$-relative equilibria of the normal form equivalent system to the periodic orbits of the original system transforms the $S^{1}$-symmetry of the normal form Hamiltonian into a $S^{1}$-symmetry of the periodic solutions of the original system. The $G \times S^{1}$-action on the $T_{\nu_{\mathrm{o}}}$-periodic solutions is defined as $(g, \theta) \cdot u(t):=g \cdot u\left(t+\frac{t \theta}{2 \pi}\right)$, where $u: \mathbb{R} \rightarrow V$ is a smooth function such that $u\left(t+T_{\nu_{\circ}}\right)=u(t)$.

The use of the isotropy subgroups of the $G \times S^{1}$-symmetry of the normal form equivalent system has been very fruitful in the symmetric bifurcation theory (see [20] for a taste of it).

In this section we will generalize Theorem 3.1 to the search of RPOs which, as solutions, have as isotropy subgroup a nontrivial subgroup of $G \times S^{1}$. Before we get into the statement and proof of this generalization we study in detail the $G \times S^{1}$-action and its subgroups, and we explain in detail what we mean by nontrivial subgroups.

All along this section we will assume that the $G$-action on the resonance space $U_{\nu_{\circ}}$ is $G$-simple, that is, $U_{\nu_{\circ}}$ contains a $G$-stable subspace which is either non absolutely irreducible or is isomorphic to the direct sum of two copies of the same absolutely irreducible representation. In the Hamiltonian symmetric framework, this hypothesis occurs generically [15. Theorem 3.3]. Under the $G$-simplicity hypothesis we have the following result whose proof can be found in [20, Proposition 7.2, page 300]:

Proposition 3.12 Let $H \subset G \times S^{1}$ be an isotropy subgroup of the $G \times S^{1}$-action on the resonance space $U_{\nu_{0}}$. Let $\pi: G \times S^{1} \rightarrow G$ be the projection on the first factor and $K:=\pi(H) \subset G$. If the $G$-action on $U_{\nu_{\circ}}$ is $G$-simple, then:

(i) $\pi: H \rightarrow K$ is an isomorphism, hence $\operatorname{dim} H=\operatorname{dim} K$.

(ii) There is a homomorphism $\theta_{H}: K \rightarrow S^{1}$ such that

$$
H=\left\{\left(k, \theta_{H}(k)\right) \in G \times S^{1} \mid k \in K\right\} .
$$

(iii) $N(H)=N_{G}(K) \times S^{1}$.

Notice that, in the language of the previous proposition, the isotropy subgroups $K$ of the $G \times S^{1}$-action on $U_{\nu_{\circ}}$ considered in Theorem 3.1 are those for which the homomorphism $\theta_{H}$ is identically zero. These are the so called spatial symmetries. The isotropy subgroups for which $\theta_{H}$ is different from zero are called spatiotemporal symmetries, and they will be the subject of this section. The homomorphism $\theta_{H}$ will be called the temporal character of $H$ and its derivative at the identity $\rho_{H}:=T_{e} \theta_{H} \in \mathfrak{k}^{*}$ the temporal velocity of $H$. The symbol $\mathfrak{k}$ denotes the Lie algebra of $K$ and $\mathfrak{k}^{*}$ its dual. The temporal velocity allows us to express the Lie algebra $\mathfrak{h}$ of $H$ in a very convenient form:

$$
\mathfrak{h}=\left\{\left(\kappa, T_{e} \theta_{H}(\kappa)\right) \in \mathfrak{k} \times \mathbb{R} \mid \kappa \in \mathfrak{k}\right\}=\left\{\left(\kappa,\left\langle\rho_{H}, \kappa\right\rangle\right) \in \mathfrak{k} \times \mathbb{R} \mid \kappa \in \mathfrak{k}\right\} .
$$

We are now in position to state a generalization of Theorem 3.1 that incorporates spatiotemporal symmetries.

Theorem 3.13 Let $\left(V, \omega, h, G, \mathbf{J}: V \rightarrow \mathfrak{g}^{*}\right)$ be a Hamiltonian system with symmetry, with $V$ a vector space, and $G$ a compact positive dimensional Lie group that acts on $V$ in a linear and canonical fashion. Suppose that $h(0)=0, \mathbf{d} h(0)=0$ (that is, the Hamiltonian vector field $X_{h}$ has an equilibrium at the origin) and that the linear Hamiltonian vector field $A:=D X_{h}(0)$ is non degenerate and contains $\pm i \nu_{\circ}$ in its spectrum. Let $U_{\nu_{\circ}}$ be the resonance space of $A$ with primitive period $T_{\nu_{\circ}}:=\frac{2 \pi}{\nu_{\circ}}$. Consider the $G \times S^{1}$-action on $U_{\nu_{\circ}}$, where the $S^{1}$-action is induced by the semisimple part of $A$, and the Lie group $G$ acts simply on $U_{\nu_{\circ}}$ Let $H=\left\{\left(k, \theta_{H}(k)\right) \mid k \in K \subset G\right\} \subset G \times S^{1}$ be an isotropy subgroup of the $G \times S^{1-}$ action on $U_{\nu_{\circ}}$ with temporal character $\theta_{H}$, temporal velocity $\rho_{H} \in \mathfrak{k}^{*}$, and such that the quadratic form $Q^{H}$ on the $H$-fixed point space $U_{\nu_{\circ}}^{H}$ defined by

$$
Q^{H}(v):=\frac{1}{2} \mathbf{d}^{2} h(0)(v, v), \quad v \in U_{\nu_{\circ}}^{H}
$$


is definite. Then, for any $\chi_{\circ} \in\left(\mathfrak{k}^{\circ}\right)^{K}$ for which $\left.\mathbf{J}\right|_{\left(U_{\nu_{\circ}}\right)_{H}} ^{-1}\left(\chi_{\circ}-\frac{1}{\nu_{\circ}} \rho_{H}\right) \cap Q_{H}^{-1}(1)$ is non empty $\left(Q_{H}:=\right.$ $\left.\left.Q^{H}\right|_{\left(U_{\nu}\right)_{H}}\right)$ there exists an open neighborhood $V_{\chi_{\circ}}$ of $\chi_{\circ}$ in $\left(\mathfrak{k}^{\circ}\right)^{K}$ such that for any $\chi \in V_{\chi_{\circ}}$, the intersection $\left.\mathbf{J}\right|_{\left(U_{\nu_{\circ}}\right)_{H}} ^{-1}\left(\chi-\frac{1}{\nu_{\circ}} \rho_{H}\right) \cap Q_{H}^{-1}(1)$ is a submanifold of $\left(U_{\nu_{\circ}}\right)_{H}$ of dimension $\operatorname{dim} U_{\nu_{\circ}}^{H}-\operatorname{dim} N(H) / H$. Suppose that the following two generic hypotheses hold:

(H1) The restriction $\left.h\right|_{U_{\nu_{\circ}}^{H}}$ of the Hamiltonian $h$ to the fixed point subspace $U_{\nu_{\circ}}^{H}$ is not radial with respect to the norm associated to $Q^{H}$.

(H2) Let $h_{k}(v):=\frac{1}{k !} \mathbf{d}^{k} h(0)\left(v^{(k)}\right), v \in U_{\nu_{\circ}}^{H}$ be the first non radial term in the Taylor expansion of $\left.h\right|_{U_{\nu_{0}}^{H}}$ around zero. We will assume that $k \geq 4$ and that the restrictions of $h_{k}$ to the submanifolds $\left.\mathbf{J}\right|_{\left(U_{\nu_{\circ}}\right)_{H}} ^{-1}\left(\chi-\frac{1}{\nu_{\circ}} \rho_{H}\right) \cap Q_{H}^{-1}(1)$, with $\chi \in V_{\chi_{\circ}}$, are $\left(N_{G}(K)_{\rho_{H}} \cap N_{G}(K)_{\chi}\right) \times S^{1}$-Morse.

Then, for any $\epsilon>0$ close enough to zero, $\chi \in V_{\chi_{\circ}}$, and $\lambda:=\Xi^{*}\left(\chi-\frac{1}{\nu_{\circ}} \rho_{H}, \frac{1}{\nu_{\circ}}\right)$, there are at least

$$
\max \left[\frac{1}{2}\left(\operatorname{dim} U_{\nu_{\circ}}^{H}-\operatorname{dim} N_{G}(K)-\operatorname{dim}\left(N_{G}(K)_{\rho_{H}} \cap N_{G}(K)_{\chi}\right)+2 \operatorname{dim} K\right), \chi_{E}\left(\mathbf{E} \mathbf{J}_{L_{H}}^{-1}(\lambda)\right)^{L_{\lambda}}\right]
$$

distinct relative periodic orbits of $X_{h}$ with energy $\epsilon$, momentum $\epsilon\left(\chi-\frac{1}{\nu_{\circ}} \rho_{H}\right) \in \mathfrak{g}^{*}$, isotropy subgroup $H$, and relative period close to $T_{\nu_{\circ}}$. By definition $\mathbf{E} \mathbf{J}_{L_{H}}^{-1}(\lambda):=\mathbf{J}_{\left(U_{\nu_{\circ}}\right)_{H}}^{-1}\left(\chi-s \rho_{H}\right) \cap Q_{A_{s}}^{-1}\left(s \nu_{\circ}\right)$ and $L_{\lambda}:=\left(\left(N_{G}(K)_{s \rho_{H}} \cap N_{G}(K)_{\chi}\right) \times S^{1}\right)$. The symbol $\chi_{E}\left(\mathbf{E J}_{L_{H}}^{-1}(\lambda)\right)^{L_{\lambda}}$ denotes the $L_{\lambda}-$ Euler characteristic of $\mathbf{E J}_{L_{H}}^{-1}(\lambda)$ (which in this case equals the standard Euler characteristic of the symplectic quotient $\left.\chi_{E}\left(\mathbf{E} \mathbf{J}_{L_{H}}^{-1}(\lambda) / L_{\lambda}\right)\right)$.

\subsection{Proof of Theorem 3.13}

In the sequel we will think of the Lie algebra $\mathfrak{k}$ and its dual $\mathfrak{k}^{*}$ as subspaces of $\mathfrak{g}$ and $\mathfrak{g}^{*}$, respectively, by choosing in $\mathfrak{g}$ an $\operatorname{Ad}_{N_{G}(K)}$-invariant inner product $\langle\cdot, \cdot\rangle$ and making $\mathfrak{g}=\mathfrak{k} \oplus \mathfrak{m}$, with $\mathfrak{m}$ the orthogonal complement to $\mathfrak{k}$ in $\mathfrak{g}$ with respect to $\langle\cdot, \cdot\rangle$. If we use the inner product dual to $\langle\cdot, \cdot\rangle$ we can write $\mathfrak{g}^{*}=\mathfrak{k}^{*} \oplus \mathfrak{m}^{*}$.

We start the proof with the following proposition that provides several important facts about the temporal velocity and its relation with the Lie algebras $\mathfrak{h}$ and $\mathfrak{k}$ of $H$ and $K$, respectively:

Proposition 3.14 Let $H \subset G \times S^{1}$ be an isotropy subgroup of the $G \times S^{1}$-action on the resonance space $U_{\nu_{\circ}}$, where the $G$-action on $U_{\nu_{\circ}}$ is $G$-simple. Let $K:=\pi(H) \subset G, \theta_{H}$ be the temporal character of $H$, and $\rho_{H}:=T_{e} \theta_{H} \in \mathfrak{k}^{*}$ be its temporal velocity. Then:

(i) $\rho_{H} \in\left(\mathfrak{k}^{*}\right)^{K}$.

(ii) $\mathfrak{h}^{\circ}=\left\{\left(-s \rho_{H}+\chi, s\right) \in \mathfrak{g}^{*} \times \mathbb{R} \mid s \in \mathbb{R}, \chi \in \mathfrak{k}^{\circ}\right\}=\left\{\left(-s \rho_{H}+\mu, s\right) \in \mathfrak{g}^{*} \times \mathbb{R} \mid s \in \mathbb{R}, \mu \in \mathfrak{m}^{*}\right\}$, where $\mathfrak{h}^{\circ}$ denotes the annihilator of $\mathfrak{h}$ in $\mathfrak{g}^{*} \times \mathbb{R}$ and $\mathfrak{k}^{\circ}$ that of $\mathfrak{k}$ in $\mathfrak{g}^{*}$.

(iii) $\left(\mathfrak{h}^{\circ}\right)^{H}=\left\{\left(-s \rho_{H}+\chi, s\right) \in \mathfrak{g}^{*} \times \mathbb{R} \mid s \in \mathbb{R}, \chi \in\left(\mathfrak{k}^{\circ}\right)^{K}\right\}$

Proof (i) It is a consequence of the fact that the temporal character $\theta_{H}$ is a homomorphism. Indeed, for any $k \in K$ and $\eta \in \mathfrak{k}$ we have that

$$
\left\langle\operatorname{Ad}_{k^{-1}}^{*} \rho_{H}, \eta\right\rangle=\left\langle\rho_{H}, \operatorname{Ad}_{k^{-1}} \eta\right\rangle=T_{e} \theta_{H} \cdot \operatorname{Ad}_{k^{-1}} \eta=\left.\frac{d}{d t}\right|_{t=0} \theta_{H}\left(k^{-1} \exp t \eta k\right)=T_{e} \theta_{H} \cdot \eta=\left\langle\rho_{H}, \eta\right\rangle .
$$

(ii) Recall that we think of $\mathfrak{k}^{*}$ as a subspace of $\mathfrak{g}^{*}$ by means of the splitting $\mathfrak{g}^{*}=\mathfrak{k}^{*} \oplus \mathfrak{m}^{*}$. By definition:

$$
\begin{aligned}
\mathfrak{h}^{\circ} & =\left\{(\eta, s) \in \mathfrak{g}^{*} \times \mathbb{R} \mid\langle\eta, \kappa\rangle+s T_{e} \theta_{H} \cdot \kappa=0, \forall \kappa \in \mathfrak{k}\right\} \\
& =\left\{(\eta, s) \in \mathfrak{g}^{*} \times \mathbb{R} \mid\left\langle\eta+s \rho_{H}, \kappa\right\rangle=0, \forall \kappa \in \mathfrak{k}\right\} \\
& =\left\{(\eta, s) \in \mathfrak{g}^{*} \times \mathbb{R} \mid \eta+s \rho \in \mathfrak{k}^{\circ}\right\} \\
& =\left\{\left(-s \rho_{H}+\chi, s\right) \in \mathfrak{g}^{*} \times \mathbb{R} \mid s \in \mathbb{R}, \chi \in \mathfrak{k}^{\circ}\right\} \\
& =\left\{\left(-s \rho_{H}+\mu, s\right) \in \mathfrak{g}^{*} \times \mathbb{R} \mid s \in \mathbb{R}, \mu \in \mathfrak{m}^{*}\right\} .
\end{aligned}
$$


(iii) It is a straightforward consequence of the definition and the use of (i).

We now study the globally Hamiltonian character of the $G \times S^{1}$-action on the resonance space $U_{\nu_{0}}$. The momentum map associated to this action is

$$
\begin{aligned}
\mathbf{E J}: U_{\nu_{\circ}} & \longrightarrow \mathfrak{g}^{*} \times \operatorname{Lie}\left(S^{1}\right)^{*} \\
v & \longmapsto\left(\left.\mathbf{J}\right|_{\nu_{\circ}}(v), \frac{1}{\nu_{\circ}} Q_{A_{s}}(v)\right),
\end{aligned}
$$

where $Q_{A_{s}}$ is the quadratic form $\frac{1}{2} \omega\left(A_{s} \cdot, \cdot\right)$ associated to the semisimple part $A_{s}$ of the linearization $A$ of the Hamiltonian vector field $X_{h}$ at the equilibrium. As a particular case of what we saw in Section 2.1] we have that the globally Hamiltonian $G \times S^{1}$-action on $U_{\nu_{0}}$ induces, for each isotropy subgroup $H \subset G \times S^{1}$ globally Hamiltonian actions of $L:=N(H) / H$ on $\left(U_{\nu_{\circ}}\right)_{H}$ and $U_{\nu_{\circ}}^{H}$ with momentum maps $\mathbf{E} \mathbf{J}_{L^{H}}: U_{\nu_{\circ}}^{H} \rightarrow \mathfrak{l}^{*}$ and $\mathbf{E} \mathbf{J}_{L_{H}}:\left(U_{\nu_{\circ}}\right)_{H} \rightarrow \mathfrak{l}^{*}$ associated to these actions given by

$$
\mathbf{E J}_{L^{H}}(v)=\Xi^{*}(\mathbf{E J}(v)), \quad \mathbf{E J}_{L_{H}}(v)=\Xi^{*}(\mathbf{E J}(v)),
$$

where $\Xi^{*}:\left(\mathfrak{h}^{\circ}\right)^{H} \rightarrow \mathfrak{l}^{*}$ is the natural $N(H) / H$-equivariant isomorphism between the $H$-fixed points in the annihilator of $\mathfrak{h}$ in $\mathfrak{g}^{*} \times \mathbb{R}$ and the dual of the Lie algebra $\mathfrak{l}^{*}$ of $N(H) / H$.

Note that since the $N(H) / H$-action on $\left(U_{\nu_{\circ}}\right)_{H}$ is free, the corresponding momentum map $\mathbf{E} \mathbf{J}_{L_{H}}$ is a submersion onto its image. Let $\Delta:\left(\mathfrak{h}^{\circ}\right)^{H} \rightarrow\left(\mathfrak{k}^{\circ}\right)^{K} \times \mathbb{R}$ be the isomorphism defined by $\Delta\left(-s \rho_{H}+\chi, s\right)=$ $(\chi, s)$. The mapping $\mathbf{E} \mathbf{J}_{N_{K}}:\left(U_{\nu_{\circ}}\right)_{H} \rightarrow\left(\mathfrak{k}^{\circ}\right)^{K} \times \mathbb{R}$ defined by $\mathbf{E} \mathbf{J}_{N_{K}}:=\left.\Delta \circ \mathbf{E}\right|_{\left(U_{\nu^{\circ}}\right)_{H}}$ is also a submersion onto its image that more specifically maps, for any $v \in\left(U_{\nu_{\circ}}\right)_{H}$, as:

$$
\mathbf{E J}_{N_{K}}(v)=\Delta \circ \mathbf{E} \mathbf{J}(v)=\Delta\left(\mathbf{J}(v), \frac{1}{\nu_{\circ}} Q_{H}(v)\right)=\left(\mathbf{J}(v)+\frac{\rho_{H}}{\nu_{\circ}} Q_{H}(v), \frac{1}{\nu_{\circ}} Q_{H}(v)\right) .
$$

The following proposition justifies the notation utilized in the statement of the theorem and provides the proof of a few facts that will be needed later on:

Proposition 3.15 We use the notation introduced in the previous paragraphs. Let $\lambda \in \mathfrak{l}^{*}$ be an element in the image of $\mathbf{E} \mathbf{J}_{L_{H}}$ such that $\lambda=\Xi^{*}\left(-s \rho_{H}+\chi, s\right)$, for some $s \in \mathbb{R}$ and some $\chi \in\left(\mathfrak{k}^{\circ}\right)^{K}$. Then:

(i) $\mathbf{E J}_{L_{H}}^{-1}(\lambda)=\mathbf{E} \mathbf{J}_{N_{K}}^{-1}(\chi, s)=\left.\mathbf{J}\right|_{\left(U_{\nu^{\circ}}\right)_{H}} ^{-1}\left(\chi-s \rho_{H}\right) \cap Q_{H}^{-1}\left(s \nu_{\circ}\right) . \mathbf{E J}_{L_{H}}^{-1}(\lambda)$ is a submanifold of $\left(U_{\nu_{\circ}}\right)_{H}$ of dimension

$$
\operatorname{dim} U_{\nu_{\circ}}^{H}-\operatorname{dim} N_{G}(K)+\operatorname{dim} K-1 .
$$

(ii) The coadjoint isotropy subgroup $L_{\lambda} \subset L$ of $\lambda \in \mathfrak{l}^{*}$ can be written as $L_{\lambda}=\left(N_{G}(K)_{s \rho_{H}} \cap N_{G}(K)_{\chi}\right) \times$ $S^{1} / H$, where $N_{G}(K)_{s \rho_{H}}$ and $N_{G}(H)_{\chi}$ are the stabilizers of $s \rho_{H} \in \mathfrak{k}^{*} \subset \mathfrak{g}^{*}$ and $\chi \in\left(\mathfrak{k}^{\circ}\right)^{K}$ with respect to the coadjoint action of $N_{G}(K)$ on $\mathfrak{g}^{*}$.

(iii) The quotient $\mathbf{E} \mathbf{J}_{L_{H}}^{-1}(\lambda) / L_{\lambda}$ is a symplectic manifold of dimension $\operatorname{dim} U_{\nu_{\circ}}^{H}-\operatorname{dim} L-\operatorname{dim} L_{\lambda}=\operatorname{dim} U_{\nu_{\circ}}^{H}-\operatorname{dim} N_{G}(K)-\operatorname{dim}\left(N_{G}(K)_{s \rho_{H}} \cap N_{G}(K)_{\chi}\right)-2+2 \operatorname{dim} K$.

(iv) Let $\chi_{\circ} \in\left(\mathfrak{k}^{\circ}\right)^{K}$ be an element in $\left(\mathfrak{k}^{\circ}\right)^{K}$ such that the set $S_{\chi_{\circ}}:=\left.\mathbf{J}\right|_{\left(U_{\nu^{\circ}}\right)_{H}} ^{-1}\left(\chi_{\circ}-\frac{1}{\nu_{\circ}} \rho_{H}\right) \cap Q_{H}^{-1}(1)$ is non empty and by part (i) a submanifold of dimension (3.20). Then, there is a neighborhood $V_{\chi_{\circ}}$ of $\chi_{\circ}$ in $\left(\mathfrak{k}^{\circ}\right)^{K}$ such that for any $\chi \in V_{\chi_{\circ}}$, the set $S_{\chi}:=\left.\mathbf{J}\right|_{\left(U_{\nu_{\circ}}\right)_{H}} ^{-1}\left(\chi-\frac{1}{\nu_{\circ}} \rho_{H}\right) \cap Q_{H}^{-1}(1)$ is also nonempty and therefore a manifold of $\left(U_{\nu_{0}}\right)_{H}$ of dimension (3.20).

Proof (i) Notice that if $\lambda=\Xi^{*}\left(-s \rho_{H}+\chi, s\right)$, then

$$
\begin{aligned}
\left.\mathbf{J}\right|_{\left(U_{\nu^{\circ}}\right)_{H}} ^{-1}\left(\chi-s \rho_{H}\right) \cap Q_{A_{s}}^{-1}\left(s \nu_{\circ}\right) & =\mathbf{E} \mathbf{J}_{N_{K}}^{-1}(\chi, s)=\left.\mathbf{E} \mathbf{J}\right|_{\left(U_{\nu^{\circ}}\right)_{H}} ^{-1}\left(\chi-s \rho_{H}, s\right)=\left.\mathbf{E} \mathbf{J}\right|_{\left(U_{\nu^{\circ}}\right)_{H}} ^{-1}\left(\left(\Xi^{*}\right)^{-1}(\lambda)\right) \\
& =\left(\left.\Xi^{*} \circ \mathbf{E} \mathbf{J}\right|_{\left(U_{\nu^{\circ}}\right)_{H}}\right)^{-1}(\lambda)=\mathbf{E} \mathbf{J}_{L_{H}}^{-1}(\lambda) .
\end{aligned}
$$


Given that $\mathbf{E} \mathbf{J}_{L_{H}}$ is the momentum map associated to a free and proper action it is a submersion and its level sets are submanifolds. Therefore (3.21) has as a corollary that $\left.\mathbf{J}\right|_{\left(U_{\nu_{0}}\right)_{H}} ^{-1}\left(\chi-s \rho_{H}\right) \cap Q_{A_{s}}^{-1}\left(s \nu_{\mathrm{o}}\right)$ is a submanifold of $\left(U_{\nu_{\circ}}\right)_{H}$ of dimension $\operatorname{dim}\left(U_{\nu_{\circ}}\right)_{H}-\operatorname{dim} N(H) / H$. Now, as $N(H)=N_{G}(K) \times S^{1}$ the expression (3.20) follows.

(ii) Since $\Xi^{*}$ is $N(H) / H$-equivariant, we have that an arbitrary element $(l, \theta) H \in N(H) / H$ is actually in the isotropy subgroup $(N(H) / H)_{\lambda}$ iff $n \cdot\left(-s \rho_{H}+\chi\right)=-s \rho_{H}+\chi$. Given that $\rho_{H} \in \mathfrak{k}^{*}, \chi \in \mathfrak{m}^{*}$, and $\mathfrak{k}^{*}$ and $\mathfrak{m}^{*}$ are $N_{G}(K)$-stable, then $n \cdot\left(-s \rho_{H}+\chi\right)=-s \rho_{H}+\chi$ iff $n \cdot\left(-s \rho_{H}\right)=-s \rho_{H}$ and $n \cdot \chi=\chi$, that is, iff $n \in N_{G}(K)_{s \rho_{H}} \cap N_{G}(K)_{\chi}$. Therefore,

$$
(N(H) / H)_{\lambda}=\left(N_{G}(K)_{s \rho_{H}} \cap N_{G}(K)_{\chi}\right) \times S^{1} / H .
$$

(iii) It is a straightforward consequence of the Marsden-Weinstein Reduction Theorem [43] and points (i) and (ii).

(iv) Let $\lambda_{\circ}=\Xi^{*}\left(\chi_{\circ}-\frac{1}{\nu_{\circ}} \rho_{H}, \frac{1}{\nu_{\circ}} \rho_{H}\right) \in \mathfrak{l}^{*}$. By (i), $S_{\chi_{\circ}}=\mathbf{E} \mathbf{J}_{L_{H}}^{-1}\left(\lambda_{\circ}\right)$. Since $\mathbf{E} \mathbf{J}_{L_{H}}$ is the momentum map corresponding to a free action on $\left(U_{\nu_{0}}\right)_{H}$ and therefore a submersion onto its image, the mapping $T_{v} \mathbf{E} \mathbf{J}_{L_{H}}: T_{v}\left(U_{\nu_{\circ}}\right)_{H} \rightarrow \mathfrak{l}^{*}$ is surjective, for any $v \in S_{\chi_{\circ}}=\mathbf{E} \mathbf{J}_{L_{H}}^{-1}\left(\lambda_{\circ}\right)$. The Local Onto Theorem (see for instance [2. Theorem 3.5.2]) implies the existence of an open neighborhood $W_{\lambda_{\circ}}$ of $\lambda_{\circ}$ in $\mathfrak{l}^{*}$ and an open neighborhood $U_{v}$ of $v$ in $\left(U_{\nu_{\circ}}\right)_{H}$ such that the mapping $\left.\mathbf{E} \mathbf{J}_{L_{H}}\right|_{U_{v}}: U_{v} \rightarrow W_{\lambda_{\circ}}$ is onto. In particular, for any $\lambda \in W_{\lambda_{\circ}}$, the level set $\mathbf{E} \mathbf{J}_{L_{H}}^{-1}(\lambda)$ is non empty and, by the submersion argument is a submanifold of $\left(U_{\nu_{\circ}}\right)_{H}$ of dimension (3.20). We now construct the open neighborhood $V_{\lambda_{\circ}}$ of $\lambda_{\circ}$ whose existence we claim in the statement of the Lemma. Firstly, the set $T$ defined by $T:=\Delta \circ\left(\Xi^{*}\right)^{-1}\left(W_{\lambda_{\circ}}\right) \subset\left(\mathfrak{k}^{\circ}\right)^{K} \times \mathbb{R}$ is an open neighborhood of $\left(\chi_{\circ}, \frac{1}{\nu_{\circ}} \rho_{H}\right)$. By the openness of $T$, there exist open neighborhoods $V_{\chi_{\circ}} \subset\left(\mathfrak{k}^{\circ}\right)^{K}$ and $W_{\frac{1}{\nu_{0}} \rho_{H}} \subset \mathbb{R}$, of $\chi_{\circ}$ and $\frac{1}{\nu_{\circ}} \rho_{H}$, respectively, such that $V_{\chi_{\circ}} \times W_{\frac{1}{\nu_{0}} \rho_{H}} \subset T$. $V_{\chi_{\circ}}$ is the neighborhood needed in the statement.

We now prove a result that constitutes the spatiotemporal analog of Lemma 3.9 Let $\widehat{h_{U_{\nu_{\circ}}^{H}}}$ be the equivalent Hamiltonian in normal form associated to $\left.h\right|_{U_{\nu_{0}}^{H}}$.

Lemma 3.16 Suppose that we are under the hypotheses of Theorem 3.13. Then, the restriction of the function $h_{k} \in C^{\infty}\left(U_{\nu_{0}}^{H}\right)$ defined by $h_{k}(u):=\frac{1}{k !} \mathbf{d}^{k} h_{U_{\nu_{0}}^{H}}(0)\left(u^{(k)}\right)$, to the level sets of the form $\left.\mathbf{J}\right|_{\left(U_{\nu_{\circ}}\right)_{H}} ^{-1}\left(\chi-\frac{1}{\nu_{\circ}} \rho_{H}\right) \cap Q_{H}^{-1}(1)$, where $\chi$ sits in $V_{\chi_{\circ}}$, the neighborhood of $\chi_{\circ} \in\left(\mathfrak{k}^{\circ}\right)^{K}$ introduced in the previous Lemma, has at least

$$
\max \left[\frac{1}{2}\left(\operatorname{dim} U_{\nu_{\circ}}^{H}-\operatorname{dim} N_{G}(K)-\operatorname{dim}\left(N_{G}(K)_{\rho_{H}} \cap N_{G}(K)_{\chi}\right)+2 \operatorname{dim} K\right), \chi_{E}\left(\mathbf{E} \mathbf{J}_{L_{H}}^{-1}(\lambda)\right)^{L_{\lambda}}\right]
$$

distinct critical orbits, where $\lambda:=\Xi^{*}\left(\chi-\frac{1}{\nu_{0}} \rho_{H}, \frac{1}{\nu_{0}}\right)$ and $\chi_{E}\left(\mathbf{E J}_{L_{H}}^{-1}(\lambda)\right)^{L_{\lambda}}$ denotes the $L_{\lambda}$-Euler characteristic of $\mathbf{E J}_{L_{H}}^{-1}(\lambda)$.

Furthermore, let $\chi^{\prime} \in V_{\chi_{\circ}} \subset\left(\mathfrak{k}^{\circ}\right)^{K}$ be arbitrary but fixed and let $\left\{u_{1}, \ldots, u_{k}\right\}$ be the set of critical points of the restriction of the function $h_{k}$ to the level set $\left.\mathbf{J}\right|_{\left(U_{\nu^{\circ}}\right)_{H}} ^{-1}\left(\chi^{\prime}-\frac{1}{\nu_{\circ}} \rho_{H}\right) \cap Q_{H}^{-1}(1)$, provided by (3.22). Then, for each $u_{i}, i \in\{1, \ldots, k\}$, there exist a neighborhood $E \subset \mathbb{R}$ of the origin in the real line, a neighborhood $V_{\chi^{\prime}} \subset V_{\lambda_{\circ}} \subset\left(\mathfrak{k}^{\circ}\right)^{K}$ of $\chi^{\prime}$ in $\left(\mathfrak{k}^{\circ}\right)^{K}$, and a smooth function $\rho: E \times V_{\chi^{\prime}} \rightarrow Q_{H}^{-1}(1)$ such that $\rho\left(0, \chi^{\prime}\right)=u_{i}$ and also, the function $v: E \times V_{\chi^{\prime}} \rightarrow U_{\nu_{\circ}}^{H}$ defined by $v(r, \chi):=r \rho(r, \chi)$ satisfies that:

(i) $v(r, \chi) \in\left(U_{\nu_{\circ}}\right)_{H}$ iff $r \neq 0$.

(ii) $Q^{H}(v(r, \chi))=r^{2}$ and $\mathbf{J}(v(r, \chi))=r^{2}\left(\chi-\frac{1}{\nu_{\circ}} \rho_{H}\right), r \in E, \chi \in V_{\chi^{\prime}}$.

(iii) $\left.\mathbf{d} \widehat{\left.h\right|_{U_{\nu_{0}}^{H}}}\right|_{\mathbf{J}_{\left(U_{\nu_{0}}\right)}^{-1}\left(r^{2}\left(\chi-\frac{1}{\nu_{\mathrm{O}}} \rho_{H}\right)\right) \cap Q_{H}^{-1}\left(r^{2}\right)}(v(r, \chi))=\left.\mathbf{d} \widehat{\left.h\right|_{U_{\nu_{0}}^{H}}}\right|_{\mathbf{E J}_{N_{K}}^{-1}\left(r^{2} \chi, \frac{r^{2}}{\nu_{0}}\right)}(v(r, \chi))=0$, that is, the branches $v(r, \chi)$ are made of critical points of the restriction of the function $\widehat{h_{U_{\nu_{\circ}}^{H}}}$ to the level sets

$$
\left.\mathbf{J}\right|_{\left(U_{\nu_{\circ}}\right)_{H}} ^{-1}\left(r^{2}\left(\chi-\frac{1}{\nu_{\circ}} \rho_{H}\right)\right) \cap Q_{H}^{-1}\left(r^{2}\right) .
$$


Proof The estimate (3.22) is a straightforward consequence of Proposition 3.15] Corollary [2.5] the statement (2.7), and the invariance properties of $h_{k}$.

We now construct the branches $v(r, \chi)$ in a fashion similar to Lemma 3.9 Let $\chi^{\prime} \in V_{\chi_{\circ}} \subset\left(\mathfrak{k}^{\circ}\right)^{K}$ be arbitrary but fixed and let $u_{0}$ be one of the critical points of the restriction of the function $h_{k}$ to the level set $\left.\mathbf{J}\right|_{\left(U_{\nu_{\mathrm{o}}}\right)_{H}} ^{-1}\left(\chi^{\prime}-\frac{1}{\nu_{\mathrm{o}}} \rho_{H}\right) \cap Q_{H}^{-1}(1)$, provided by (3.22). Since by Proposition 3.15 any element of the form $\left(\chi, \frac{1}{\nu_{0}}\right)$ with $\chi \in V_{\chi_{\circ}}$ is a regular value of $\mathbf{E} \mathbf{J}_{N_{K}}$, there exist neighborhoods $V_{\chi^{\prime}} \subset V_{\chi_{\circ}}$ of $\chi^{\prime}, W_{\frac{1}{\nu_{\circ}}}$ of $\frac{1}{\nu_{\circ}}$ in $\mathbb{R}, W$ of the origin in $\mathbb{R}^{s}$, with $s=\operatorname{dim} U_{\nu_{\circ}}^{H}-\operatorname{dim}\left(\mathfrak{k}^{\circ}\right)^{K}-1$, and a mapping $\psi: V_{\chi^{\prime}} \times W \times W_{\frac{1}{\nu_{0}}} \rightarrow U_{\nu_{\circ}}^{H}$ which is a diffeomorphism onto its image. The mapping $\psi$ satisfies that $\psi\left(\chi^{\prime}, 0, \frac{1}{\nu_{0}}\right)=u_{0}$ and $\mathbf{E J}_{N_{K}}(\psi(\chi, w, l))=(\chi, l)$, which is equivalent to having that $\mathbf{J}(\psi(\chi, w, l))=$ $\chi-l \rho_{H}$ and $Q_{H}(\psi(\chi, w, l))=l \nu_{\circ}$. Let $\varphi: V_{\chi^{\prime}} \times W \rightarrow Q_{H}^{-1}(1)$ be the mapping defined by $(\chi, w) \longmapsto$ $\psi\left(\chi, w, \frac{1}{\nu_{0}}\right)$. This map constitutes a local chart around the point $u_{0}$ in $Q_{H}^{-1}(1)$. Exactly as we did in Lemma 3.9 we can factor out the $\left(N_{G}(K)_{\rho_{H}} \cap N_{G}(K)_{\chi^{\prime}}\right) \times S^{1}$-action in this chart which allows us to prove the Lemma by mimicking what we did in the proof of Lemma 3.9 starting from expression (3.6).

Let now $\chi^{\prime} \in V_{\chi_{\circ}}$ and $v: E \times V_{\chi^{\prime}} \rightarrow U_{\nu_{\circ}}^{H}$ be one of the branches introduced in Lemma 3.16 By construction,

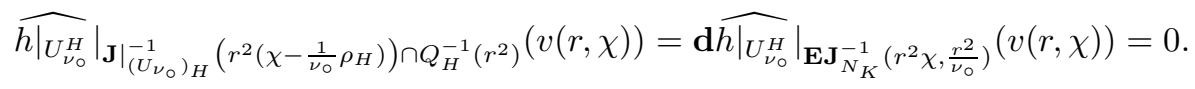

Since $\mathbf{E} \mathbf{J}_{N_{K}}$ maps into $\left(\mathfrak{k}^{\circ}\right)^{K} \times \mathbb{R}$, the Lagrange Multipliers Theorem guarantees the existence of an element $\Lambda(r, \chi) \in\left(\left(\mathfrak{k}^{\circ}\right)^{K}\right)^{*}$ and $c(r, \chi) \in \mathbb{R}$ such that

$$
\mathbf{d} \widehat{\left.h\right|_{U_{\nu_{0}}^{H}}}(v(r, \lambda))=\left[c(r, \lambda)+\left\langle\rho_{H}, \Lambda(r, \lambda)\right\rangle\right] \mathbf{d} Q^{H}(v(r, \lambda))+\mathbf{d}\left(\left.\mathbf{J}\right|_{U_{\nu_{0}}^{H}}\right)^{\Lambda(r, \lambda)}(v(r, \lambda)),
$$

which implies that $v(r, \lambda)$ is a periodic point of the Hamiltonian vector field $X_{\widehat{\left.h\right|_{\mathcal{V}_{\circ}}}}-\left(\mathbf{J}_{U_{\mathcal{V}_{0}}^{H}}\right)^{\Lambda(r, \lambda)}$. If we are able to prove that $\Lambda(r, \lambda)$ becomes very small as $r \rightarrow 0$, the Normal Form Theorem will guarantee that $v(r, \lambda)$ will amount to a periodic point of $X_{\left.h\right|_{U_{\nu_{0}}^{H}}-\left(\mathbf{J}_{U_{\nu_{0}}^{H}}\right)^{\Lambda(r, \lambda)}}=X_{\left.h\right|_{U_{\nu_{0}}^{H}}}-(\Lambda(r, \lambda))_{U_{\nu_{0}}^{H}}$, that is, a RPO of $X_{h}$ (the symbol $(\Lambda(r, \lambda))_{U_{\nu_{0}}^{H}}$ denotes the vector field defined by $(\Lambda(r, \lambda))_{U_{\nu_{0}}^{H}}(v)=\left.\frac{d}{d t}\right|_{t=0} \exp t \Lambda(r, \lambda)$. $v, v \in U_{\nu_{0}}^{H}$. It is easy to show that this is a well defined vector field on $U_{\nu_{\circ}}^{H}$ since $\exp t \Lambda(r, \lambda) \cdot v \in$ $U_{\nu_{\circ}}^{H}$ whenever $v \in U_{\nu_{\circ}}^{H}$ ). Actually it can be easily proved by mimicking what we did in the proof of Theorem [3.1] after (3.11), that $\Lambda(r, \chi)$ and $c(r, \chi)$ are smooth functions that tend to zero and one, respectively, as the variable $r$ tends to zero.

Remark 3.17 As we already pointed out in the introduction Theorem 3.13 is NOT a generalization of Theorem 3.1] In the proof of Theorem [3.1 intervenes a transversality argument that guarantees that all the solutions obtained are genuine RPOs and not just relative equilibria. This is explicitly mentioned in its statement. Even though the spatial symmetries in the relative periodic solutions predicted in Theorem 3.1 are a particular case of the spatiotemporal symmetries treated in Theorem 3.13 one does not generalize the other since the subgroups of $G \times S^{1}$ intertwine the $G$ and $S^{1}$-actions via the temporal character preventing us from making the distinction between RPOs and relative equilibria. In short, we cannot guarantee that the RPOs provided by Theorem 3.13 are not just relative equilibria.

\section{Relative periodic orbits around stable relative equilibria}

In this section we will use the so called Marle-Guillemin-Sternberg (MGS) normal form and the reconstruction equations in order to generalize the main result in the previous section to the search of RPOs around genuine relative equilibria. 


\subsection{The MGS normal form and the reconstruction equations}

Since this topic has been already introduced already in many other papers we will just briefly sketch the results that we will need in our exposition, and will leave the reader interested in the details consult the original papers [41, 23]. Regarding the reconstruction equations the reader is encouraged to check with [52, 60, 55].

All along this section we will work with a $G$-Hamiltonian system $(M, \omega, h, G, \mathbf{J})$, where the Lie group $G$ acts in a proper and globally Hamiltonian fashion on the manifold $M$. Let $m$ be a point in $M$ such that $\mathbf{J}(m)=\mu \in \mathfrak{g}^{*}$ and $G_{m}$ denotes the isotropy subgroup of the point $m$. We denote by $\mathfrak{g}_{\mu}$ the Lie algebra of the stabilizer $G_{\mu}$ of $\mu \in \mathfrak{g}^{*}$ under the coadjoint action of $G$ on $\mathfrak{g}^{*}$. We now choose in ker $T_{m} \mathbf{J}$ a $G_{m}$-invariant inner product, $\langle\cdot, \cdot\rangle$, always available by the compactness of $G_{m}$. Using this inner product we define the symplectic normal space $V_{m}$ at $m \in M$ with respect to the inner product $\langle\cdot, \cdot\rangle$, as the orthogonal complement of $T_{m}\left(G_{\mu} \cdot m\right)$ in $\operatorname{ker} T_{m} \mathbf{J}$, that is, $\operatorname{ker} T_{m} \mathbf{J}=T_{m}\left(G_{\mu} \cdot m\right) \oplus V_{m}$, where the symbol $\oplus$ denotes orthogonal direct sum. It is easy to verify that $\left(V_{m},\left.\omega(m)\right|_{V_{m}}\right)$ is a $G_{m}$-invariant symplectic vector space. Let $B^{\sharp}: V_{m}^{*} \rightarrow V_{m}$ be the isomorphism associated to the symplectic form $\left.\omega(m)\right|_{V_{m}}$

Recall that by the equivariance of $\mathbf{J}$, the isotropy subgroup $G_{m}$ of $m$ is a subgroup of $G_{\mu}$ and therefore $\mathfrak{g}_{m}=\operatorname{Lie}\left(G_{m}\right) \subset \mathfrak{g}_{\mu}$. Using again the compactness of $G_{m}$, we construct an inner product $\langle\cdot, \cdot\rangle$ on $\mathfrak{g}$, invariant under the restriction to $G_{m}$ of the adjoint action of $G$ on $\mathfrak{g}$. Relative to this inner product we can write the following orthogonal direct sum decompositions $\mathfrak{g}=\mathfrak{g}_{\mu} \oplus \mathfrak{q}$, and $\mathfrak{g}_{\mu}=\mathfrak{g}_{m} \oplus \mathfrak{m}$, for some subspaces $\mathfrak{q} \subset \mathfrak{g}$ and $\mathfrak{m} \subset \mathfrak{g}_{\mu}$. The inner product also allows us to identify all these Lie algebras with their duals. In particular, we have the dual orthogonal direct sums $\mathfrak{g}^{*}=\mathfrak{g}_{\mu}^{*} \oplus \mathfrak{q}^{*}$ and $\mathfrak{g}_{\mu}^{*}=\mathfrak{g}_{m}^{*} \oplus \mathfrak{m}^{*}$ which allow us to consider $\mathfrak{g}_{\mu}^{*}$ as a subspace of $\mathfrak{g}^{*}$ and, similarly, $\mathfrak{g}_{m}^{*}$ and $\mathfrak{m}^{*}$ as subspaces of $\mathfrak{g}_{\mu}^{*}$.

The $G_{m}$-invariance of the inner product utilized to construct the splittings $\mathfrak{g}_{\mu}=\mathfrak{g}_{m} \oplus \mathfrak{m}$ and $\mathfrak{g}_{\mu}^{*}=\mathfrak{g}_{m}^{*} \oplus \mathfrak{m}^{*}$, implies that both $\mathfrak{m}$ and $\mathfrak{m}^{*}$ are $G_{m}$-spaces using the restriction to them of the $G_{m}-$ adjoint and coadjoint actions, respectively.

The importance of all these objects is in the fact that there is a positive number $r>0$ such that, denoting by $\mathfrak{m}_{r}^{*}$ the open ball of radius $r$ relative to the $G_{m}$-invariant inner product on $\mathfrak{m}^{*}$, the manifold $Y_{r}:=G \times_{G_{m}}\left(\mathfrak{m}_{r}^{*} \times V_{m}\right)$ can be endowed with a symplectic structure $\omega_{Y_{r}}$ with respect to which the left $G$-action $g \cdot[h, \eta, v]=[g h, \eta, v]$ on $Y_{r}$ is globally Hamiltonian with Ad $^{*}$-equivariant momentum map $\mathbf{J}_{Y_{r}}: Y_{r} \rightarrow \mathfrak{g}^{*}$ given by $\mathbf{J}_{Y_{r}}([g, \rho, v])=\operatorname{Ad}_{g^{-1}}^{*} \cdot\left(\mu+\rho+\mathbf{J}_{V_{m}}(v)\right)$. Moreover, there exist $G$-invariant neighborhoods $U$ of $m$ in $M, U^{\prime}$ of $[e, 0,0]$ in $Y_{r}$, and an equivariant symplectomorphism $\phi: U \rightarrow U^{\prime}$ satisfying $\phi(m)=[e, 0,0]$ and $\mathbf{J}_{Y_{r}} \circ \phi=\mathbf{J}$. On other words, the twisted product $Y_{r}$ can be used as a coordinate system in a tubular neighborhood of the orbit $G \cdot m$. This semi-global coordinates are referred to as the MGS normal form.

In what follows we will use the MGS coordinates to compute the equations that describe the dynamics induced by the Hamiltonian vector field corresponding to a $G$-invariant Hamiltonian. These are called the bundle 60 or reconstruction 52 equations. Let $h \in C^{\infty}(Y)^{G}$ be a $G$-invariant Hamiltonian on $Y$. Our aim is to compute the differential equations that determine the $G$-equivariant Hamiltonian vector field $X_{h} \in \mathfrak{X}(Y)$ associated to $h$ and characterized by $\mathbf{i}_{X_{h}} \omega_{Y}=\mathbf{d} h$.

Since the projection $\pi: G \times \mathfrak{m}^{*} \times V_{m} \rightarrow G \times_{H}\left(\mathfrak{m}^{*} \times V_{m}\right)$ is a surjective submersion, there are always local sections available that we can use to locally express $X_{h}=T \pi\left(X_{G}, X_{\mathfrak{m}^{*}}, X_{V_{m}}\right)$, with $X_{G}, X_{\mathfrak{m}^{*}}$ and $X_{V_{m}}$ locally defined smooth maps on $Y$ and having values in $T G, T \mathfrak{m}^{*}$ and $T V_{m}$ respectively. Thus, for any $[g, \rho, v] \in Y$, one has $X_{G}([g, \rho, v]) \in T_{g} G, X_{\mathfrak{m}^{*}}([g, \rho, v]) \in T_{\rho} \mathfrak{m}^{*}=\mathfrak{m}^{*}$, and $X_{V_{m}}([g, \rho, v]) \in$ $T_{v} V_{m}=V_{m}$. Moreover, using the $\operatorname{Ad}_{G_{m}}$-invariant decomposition of the Lie algebra $\mathfrak{g}: \mathfrak{g}=\mathfrak{g} m \oplus \mathfrak{m} \oplus$ $\mathfrak{q}$, the mapping $X_{G}$ can be written, for any $[g, \rho, v] \in Y$, as $X_{G}([g, \rho, v])=T_{e} L_{g}\left(X_{\mathfrak{g}_{m}}([g, \rho, v])+\right.$ $\left.X_{\mathfrak{m}}([g, \rho, v])+X_{\mathfrak{q}}([g, \rho, v])\right)$, with $X_{\mathfrak{g}_{m}}, X_{\mathfrak{m}}$, and $X_{\mathfrak{q}}$, locally defined smooth maps on $Y$ with values in $\mathfrak{g}_{m}, \mathfrak{m}$, and $\mathfrak{q}$ respectively. Also, note that since $h \in C^{\infty}\left(G \times{ }_{H}\left(\mathfrak{m}^{*} \times V_{m}\right)\right)^{G}$ is $G$-invariant, the mapping $h \circ \pi \in C^{\infty}\left(G \times \mathfrak{m}^{*} \times V_{m}\right)^{H}$ can be understood as a $H$-invariant function that depends only on the $\mathfrak{m}^{*}$ and $V_{m}$ variables, that is, $h \circ \pi \in C^{\infty}\left(\mathfrak{m}^{*} \times V_{m}\right)^{H}$.

Using these ideas and the explicit expression of the symplectic form $\omega_{Y_{r}}$ we can explicitly write down the differential equations that determine the components of $X_{h}$. In order to do so we first implicitly define a function $\eta: \mathfrak{g}_{\mu} \times \mathfrak{q} \rightarrow \mathfrak{q}^{*}$ such that $\eta(\xi, 0)=0$ for all $\xi \in \mathfrak{g}_{\mu}$ and that for $\rho \in \mathfrak{m}^{*}$, $v \in V_{m}$ small enough satisfies $\mathbb{P}_{\mathfrak{q}^{*}}\left(\operatorname{ad}_{\left(X_{\mathfrak{m}}+\eta\left(X_{\mathfrak{m}}, \rho+\mathbf{J}_{V_{m}}(v)\right)\right)}\left(\rho+\mathbf{J}_{V_{m}}(v)+\mu\right)\right)=0$. If we define $\psi(\rho, v):=$ 
$\eta\left(D_{\mathfrak{m}^{*}}(h \circ \pi), \rho+\mathbf{J}_{V_{m}}(v)\right)$, then:

$$
\begin{gathered}
X_{\mathfrak{g}_{m}}=0 \\
X_{\mathfrak{q}}=\psi(\rho, v) \\
X_{\mathfrak{m}}=D_{\mathfrak{m}^{*}}(h \circ \pi) \\
X_{V_{m}}=B_{V_{m}}^{\sharp}\left(D_{V_{m}}(h \circ \pi)\right) \\
X_{\mathfrak{m}^{*}}=\mathbb{P}_{\mathfrak{m}^{*}}\left(\operatorname{ad}_{D_{\mathfrak{m} *}(h \circ \pi)}^{*} \rho+\operatorname{ad}_{D_{\mathfrak{m}^{*}}(h \circ \pi)}^{*} \mathbf{J}_{V_{m}}(v)+\operatorname{ad}_{\psi(\rho, v)}^{*}\left(\rho+\mathbf{J}_{V_{m}}(v)\right)\right) .
\end{gathered}
$$

The previous equations admit severe simplifications in the presence of various Lie algebraic hypotheses. See [60] for an extensive study. For future reference we will note two particularly important cases:

- The Lie algebra $\mathfrak{g}$ is Abelian: in that case $X_{\mathfrak{m}^{*}}=X_{\mathfrak{q}}=0$ at any point.

- The point $\mu \in \mathfrak{g}^{*}$ is split [21, that is, the Lie algebra $\mathfrak{g}_{\mu}$ of the coadjoint isotropy of $\mu$ admits a $\operatorname{Ad}_{G_{\mu}}$-invariant complement in $\mathfrak{g}$ : in that case the mappings $\eta$ and $\psi$ are identically zero.

\subsection{The main estimate}

The following result generalizes theorems 3.1 and 3.13 to the search of RPOs around stable relative equilibria

Theorem 4.1 Let $(M, \omega, h, G, \mathbf{J})$ be a Hamiltonian $G$-space. Let $m \in M$ be a relative equilibrium of this system with velocity $\xi \in \mathfrak{g}$, isotropy $G_{m}, \mathbf{J}(m)=\mu \in \mathfrak{g}^{*}$, and $h(m)=0$. Let $V_{m} \subset T_{m} M$ be any symplectic normal space through the point $m, Q_{V_{m}}:=\left.\frac{1}{2} \mathbf{d}^{2}\left(h-\mathbf{J}^{\mathbb{P}_{\mathrm{m}} \xi}\right)(m)\right|_{V_{m}}$, and $A_{V_{m}}:=X_{Q_{V_{m}}}$. Suppose that for $V_{m}$ (and hence for any other symplectic normal space) the infinitesimally symplectic linear map $A_{V_{m}}$ is non singular and has $\pm i \nu_{\circ}$ as eigenvalues. Let $U_{\nu_{\circ}}$ be the $G_{m} \times S^{1}$-symmetric resonance space of $A_{V_{m}}$ with primitive period $T_{\nu_{\circ}}$ (the $S^{1}$-action is generated by the semisimple part of the restriction of $A_{V_{m}}$ to $U_{\nu_{0}}$ ). Let $H \subset G_{m} \times S^{1}$ be an isotropy subgroup of the $G_{m} \times S^{1}$-action on $U_{\nu_{\circ}}$. If $H$ is not a purely spatial subgroup of $G_{m} \times S^{1}$ we assume that the $G_{m}$-action on $U_{\nu_{\circ}}$ is simple, and hence it has an associated temporal character $\theta_{H}$ such that

$$
H=\left\{\left(f, \theta_{H}(f)\right) \mid f \in K \subset G_{m}\right\}
$$

and a well defined temporal velocity $\rho_{H}:=T_{e} \theta_{H} \in \mathfrak{k}^{*}$.

Suppose that $\left.\mathbf{d}^{2}\left(h-\mathbf{J}^{\mathbb{P}_{\mathfrak{m}} \xi}\right)(m)\right|_{U_{\nu_{0}}^{H}}$ is a definite quadratic form. We consider two cases:

(i) If $\rho_{H}=0$ and $\left.\mathbf{J}_{V_{m}}\right|_{\left(U_{\nu_{0}}\right)_{H}} ^{-1}(0) \cap Q_{V_{m}}^{-1}(1)$ is nonempty then, for any $\epsilon>0$ small enough, there exist generically

$$
\max \left[\frac{1}{2}\left(\operatorname{dim} U_{\nu_{\circ}}^{H}-2 \operatorname{dim} N_{G_{m}}(K)+2 \operatorname{dim} K\right), \chi_{E}\left(\left.\mathbf{J}_{V_{m}}\right|_{\left(U_{\nu^{\circ}}\right)_{H}} ^{-1}(0) \cap Q_{V_{m}}^{-1}(1)\right)^{N_{G_{m}}(K) \times S^{1}}\right]
$$

distinct RPOs of $X_{h}$ with energy $\epsilon$, momentum $\mu \in \mathfrak{g}^{*}$, relative period close to $T_{\nu_{\mathrm{o}}}$, and isotropy subgroup $G_{m}$.

(ii) If $\rho_{H} \neq 0$ and $\chi_{\circ} \in\left(\mathfrak{k}^{\circ}\right)^{K}$ is such that $\left.\mathbf{J}_{V_{m}}\right|_{\left(U_{\nu^{\circ}}\right)_{H}} ^{-1}\left(\chi_{\circ}-\frac{1}{\nu_{\circ}} \rho_{H}\right) \cap Q_{V_{m}}^{-1}(1)$ is non empty, assume that ONE of the following hypotheses holds:

1. The Lie algebra $\mathfrak{g}$ is Abelian. 
2. The Lie algebra $\mathfrak{g}_{\mu}$ is Abelian and $\mu$ is split.

3. $\mathfrak{g}_{m}=\mathfrak{g}_{\mu}$.

Then, there exists an open neighborhood $V_{\chi_{\circ}}$ of $\chi_{\circ}$ in $\left(\mathfrak{k}^{\circ}\right)^{K}$ such that for any $\chi \in V_{\chi_{\circ}}$, the intersection $\left.\mathbf{J}_{V_{m}}\right|_{\left(U_{\nu^{\circ}}\right)_{H}} ^{-1}\left(\chi-\frac{1}{\nu_{\circ}} \rho_{H}\right) \cap Q_{V_{m}}^{-1}(1)$ is a submanifold of $\left(U_{\nu_{\circ}}\right)_{H}$ of dimension $\operatorname{dim} U_{\nu_{\circ}}^{H}-$ $\operatorname{dim} N_{G_{m} \times S^{1}}(H) / H$. Moreover, for any $\epsilon>0$ close enough to zero and $\chi \in V_{\chi_{0}}$, there are generically at least

$$
\begin{gathered}
\max \left[\frac{1}{2}\left(\operatorname{dim} U_{\nu_{\circ}}^{H}-\operatorname{dim} N_{G_{m}}(K)-\operatorname{dim}\left(N_{G_{m}}(K)_{\rho_{H}} \cap N_{G_{m}}(K)_{\chi}\right)+2 \operatorname{dim} K\right),\right. \\
\left.\chi_{E}\left(\left.\mathbf{J}_{V_{m}}\right|_{\left(U_{\nu_{\circ}}\right)_{H}} ^{-1}\left(\chi-\frac{1}{\nu_{\circ}} \rho_{H}\right) \cap Q_{V_{m}}^{-1}(1)\right)^{\left(N_{G_{m}}(K)_{\rho_{H}} \cap N_{G_{m}}(K)_{\chi}\right) \times S^{1}}\right]
\end{gathered}
$$

distinct relative periodic orbits of $X_{h}$ with energy $\epsilon$, momentum $\mu+\epsilon\left(\chi-\frac{1}{\nu_{\circ}} \rho_{H}\right) \in \mathfrak{g}^{*}$, isotropy $H$, and relative period close to $T_{\nu_{0}}$.

The symbol $\mathbf{J}_{V_{m}}$ denotes the momentum map associated to the linear action of $G_{m}$ on $V_{m}$. The projections $\mathbb{P}_{\mathfrak{g}_{m}}$ and $\mathbb{P}_{\mathfrak{m}}$ are consistent with a given $\operatorname{Ad}_{G_{m}}$-invariant splitting $\mathfrak{g}=\mathfrak{g}_{m} \oplus \mathfrak{m} \oplus \mathfrak{q}$ of the Lie algebra $\mathfrak{g}$.

Remark 4.2 The reader interested in the relation between the hypothesis on the definiteness of the quadratic form $\left.\mathbf{d}^{2}\left(h-\mathbf{J}^{\mathbb{P}_{\mathfrak{m}} \xi}\right)(m)\right|_{V_{m}}$ and the actual nonlinear stability of the relative equilibrium is encouraged to check with [35] 54, 59, and references therein.

Proof of the Theorem A straightforward computation shows that the Hessian in the statement of the theorem is well defined and that the hypotheses on it do not depend on the choice of symplectic normal space $V_{m}$.

Given the local nature of the statement, we can use the MGS coordinates to carry out the proof of the theorem. For simplicity in the exposition we will identify points and maps in $M$ and their counterparts in the MGS coordinates $Y$. Those coordinates can be chosen so that the point $m$ is represented by $[e, 0,0] \in G \times_{G_{m}}\left(\mathfrak{m}^{*} \times V_{m}\right)$ and the submanifold $\Sigma_{m}:=\{e\} \times_{G_{m}}\left(\{0\} \times V_{m}\right) \subset Y$ is such that $T_{m} \Sigma_{m}$ is a symplectic normal space at $m$, that is, $\operatorname{ker} T_{m} \mathbf{J}=T_{m} \Sigma_{m} \oplus T_{m}\left(G_{\mu} \cdot m\right)$.

Notice that in MGS coordinates the point $m \equiv[e, 0,0]$ is a relative equilibrium of the Hamiltonian vector field $X_{h}$ with velocity $\xi$ when

$$
X_{h}(m)=T_{(e, 0,0)} \pi(\xi, 0,0)
$$

and the associated flow behaves as $F_{t}(m)=[\exp t \xi, 0,0]$.

We now define the function $h_{V_{m}} \in C^{\infty}\left(V_{m}\right)^{G_{m}}$ as $h_{V_{m}}(v)=h \circ \pi(0, v)$, for each $v \in V_{m}$. Moreover, notice that by (4.8) and the reconstruction equation (4.4)

$$
\mathbf{d} h_{V_{m}}(0)=D_{V_{m}}(h \circ \pi)(0,0)=B_{V_{m}}^{b}\left(X_{V_{m}}(0,0,0)\right)=0,
$$

where $B \in \Lambda^{2}\left(V_{m} \times V_{m}\right)$ is the Poisson tensor associated to the symplectic form $\omega_{V_{m}}:=\left.\omega\right|_{V_{m}}$. Also, for any $v, w \in V_{m}$ :

$$
\begin{array}{r}
\mathbf{d}^{2}\left(h-\mathbf{J}^{\mathbb{P}_{\mathfrak{m}} \xi}\right)([e, 0,0])\left(T_{(e, 0,0)} \pi(0,0, v), T_{(e, 0,0)} \pi(0,0, w)\right)=\left.\left.\frac{d}{d t}\right|_{t=0} \frac{d}{d s}\right|_{s=0}\left(h-\mathbf{J}^{\mathbb{P}_{\mathfrak{m}} \xi}\right)([e, 0, t v+s w]) \\
=\mathbf{d}^{2} h_{V_{m}}(0)(v, w)-\left.\frac{d}{d t}\right|_{t=0}\left\langle T_{t v} \mathbf{J}_{V_{m}} \cdot w, \mathbb{P}_{\mathfrak{m}} \xi\right\rangle=\mathbf{d}^{2} h_{V_{m}}(0)(v, w),
\end{array}
$$

since $T_{t v} \mathbf{J}_{V_{m}} \cdot w \in \mathfrak{g}_{m}^{*}$ for any $t$. Therefore, the hypothesis on the non degeneracy of $\left.\mathbf{d}^{2}\left(h-\mathbf{J}^{\mathbb{P}_{\mathfrak{m}} \xi}\right)(m)\right|_{U_{\nu}}$ implies the non degeneracy of $\left.\mathbf{d}^{2} h_{V_{m}}(0)\right|_{U_{\nu}}$. 
We now apply Theorem 3.13 to the equilibrium that the system $\left(V_{m}, \omega_{V_{m}}, h_{V_{m}}, G_{m}, \mathbf{J}_{V_{m}}\right)$ has at the origin. If we use isotropy subgroups of the $G_{m} \times S^{1}$-action on $V_{m}$ with temporal velocity equal to zero and look for RPOs such that $\chi=0$, Theorem 3.13 provides us with (4.6) RPOs for $\left(V_{m}, \omega_{V_{m}}, h_{V_{m}}, G_{m}, \mathbf{J}_{V_{m}}\right)$ with $\mathbf{J}_{V_{m}}$ momentum equal to zero. The general case with non zero temporal character and arbitrary $\chi$ gives us (4.7) relatively periodic solutions with $\mathbf{J}_{V_{m}}$ momentum equal to $\epsilon\left(\chi-\frac{1}{\nu_{0}} \rho_{H}\right)$.

In the remainder of the proof we will use these $G_{m}$-relative periodic orbits in $V_{m}$ to construct $G-$ relative periodic orbits in the original system using the reconstruction equations. We will first establish the estimate (4.6) on the number of RPOs with momentum equal to $\mu$ : let $v \in V_{m}$ be one of the $G_{m}-$ relative periodic orbits of $\left(V_{m}, \omega_{V_{m}}, h_{V_{m}}, G_{m}, \mathbf{J}_{V_{m}}\right)$ with $\mathbf{J}_{V_{m}}$ momentum equal to zero. If we look at the reconstruction equation (4.5) taking into account that $\mathbf{J}_{V_{m}}(v)=0$ we obtain that the point $[e, 0, v]$ is such that $X_{\mathfrak{m}}^{*}([e, 0, v])=0$ and therefore it is necessarily a $G$-relative periodic point of $X_{h}$. Notice that, given the expression of the momentum map in MGS coordinates, this RPO has momentum exactly equal to $\mu$.

As to the estimate (4.7), consider now one of the RPOs of $\left(V_{m}, \omega_{V_{m}}, h_{V_{m}}, G_{m}, \mathbf{J}_{V_{m}}\right)$ with $\mathbf{J}_{V_{m}}$ momentum equal to $\epsilon\left(\chi-\frac{1}{\nu_{\circ}} \rho_{H}\right)$. Additionally, suppose that we are in any of the first two cases contemplated in the Lie algebraic hypotheses in the statement of the theorem, that is, either the Lie algebra $\mathfrak{g}$ is Abelian or $\mathfrak{g}_{\mu}$ is Abelian and $\mu$ is split. It is easy to see by looking at the reconstruction equation (4.5) that in any of those two cases $X_{\mathfrak{m}}^{*}=0$ at any point and therefore if $v \in V_{m}$ is one of the $G_{m}-\mathrm{RPOs}$ of $\left(V_{m}, \omega_{V_{m}}, h_{V_{m}}, G_{m}, \mathbf{J}_{V_{m}}\right)$ the point $[e, 0, v]$ is necessarily a $G-\mathrm{RPO}$ of the original system, with $G-$ momentum map $\mu+\epsilon\left(\chi-\frac{1}{\nu_{\circ}} \rho_{H}\right) \in \mathfrak{g}^{*}$ and isotropy subgroup $H$. If we are under hypothesis 3 , the fact that $\mathfrak{g}_{m}=\mathfrak{g}_{\mu}$ implies that $\mathfrak{m}^{*}=0$ and therefore the argument that we just used can be applied to the points of the form $[e, v]$.

Conclusions. In this paper we have proved results that give estimates on the number of relative periodic orbits around given stable equilibria and relative equilibria.

The approach taken in the proofs carries in its wake some limitations in our results. For instance, discrete symmetries are invisible by the momentum map. It is our belief that results in this direction can only be obtained by taking a global variational approach that the author is already studying and that will be the subject of a future work.

This global variational approach seems also to be the only way to obtain global generalizations of the local results stated throughout the paper, similar to those obtained in the past regarding the Weinstein-Moser Theorem (see for instance [17, 3, 25], an references therein) where, by substituting the stability condition by convexity hypotheses, estimates regarding the existence of periodic orbits could be formulated for any convex energy level set.

Acknowledgments. I am thankful to J. Marsden for emphasizing to me the importance of the so called Smale Program that, at some level, played an important inspirational role in this work. Special thanks go also to J. Montaldi for his help and for his organizational skills that made the summer school MASESS 2000 at Peyresq (France) a comfortable place where I developed some ideas that improved this paper. I also thank A. Weinstein for kindly answering my questions, B. Ẑhilinskií for his interest and suggestions, and T. Ratiu for his encouragement. The valuable comments of an anonymous referee are also gratefully acknowledged since they had a very positive impact in this paper. This research was partially supported by the European Commission through funding for the Research Training Network Mechanics and Symmetry in Europe (MASIE).

\section{References}

[1] Abraham, R., and Marsden, J.E. [1978] Foundations of Mechanics. Second edition, Addison-Wesley.

[2] Abraham, R., Marsden, J.E., and Ratiu, T.S. [1988] Manifolds, Tensor Analysis, and Applications. Volume 75 of Applied Mathematical Sciences, Springer-Verlag.

[3] Ambrosetti, A. and Mancini, G. [1982] On a theorem by Ekeland and Lasry concerning the number of periodic Hamiltonian trajectories. J. Differential Equations, 43, 249-256. 
[4] Arms, J. M., Cushman, R., and Gotay, M.J. [1991] A universal reduction procedure for Hamiltonian group actions. In The Geometry of Hamiltonian Systems. T. S. Ratiu ed. pages 33-51. Springer Verlag.

[5] Atiyah, M. F. and Bott, R. [1982] The Yang-Mills equations over Riemann surfaces. Phil. Trans. R. Soc. Lond. A, 308, 523-615.

[6] Bartsch, T. [1994] Topological Methods for Variational Problems with Symmetries. Springer Lecture Notes in Mathematics, vol. 1560.

[7] Bartsch, T. [1997] A generalization of the Weinstein-Moser theorems on periodic orbits of a Hamiltonian system near an equilibrium. Ann. Inst. H. Poincaré Anal. Non Linéaire, 14(6), 691-718.

[8] Bates, L. and Lerman, E. [1997] Proper group actions and symplectic stratified spaces. Pacific J. Math., 181(2), 201-229.

[9] Bredon, G.E. [1972] Introduction to Compact Transformation Groups. Academic Press.

[10] Chevalley, C. [1946] Theory of Lie Groups. Princeton University Press.

[11] Chossat, P., Lewis, D., Ortega, J.-P., and Ratiu, T. S. [2002] Bifurcation of relative equilibria in mechanical systems with symmetry. To appear in Advances in Applied Mathematics.

[12] Clapp, M., and Puppe, D. [1986] Invariants of Lusternik-Schnirelmann type and the topology of critical sets. Transactions Amer. Math. Soc., 298, 603-620.

[13] Clapp, M., and Puppe, D. [1991] Critical point theory with symmetries. J. reine. angew. Math., 418, 1-29.

[14] Cushman, R.H. and Bates, L.M. [1997] Global Aspects of Classical Integrable Systems. Birkhäuser Verlag.

[15] Dellnitz, M., Melbourne, I., and Marsden, J. E. [1992] Generic Bifurcation of Hamiltonian vector fields with symmetry. Nonlinearity, 5, 979-996.

[16] Duistermaat, J.J. and Kolk, J.A. [1999] Lie Groups. Universitext, Springer-Verlag.

[17] Ekeland, I., and Lasry, J.-M. [1980] On the number of periodic trajectories for a Hamiltonian flow on a convex energy surface. Ann. Math., 112, 283-319.

[18] Fadell, E. [1985] The equivariant Lusternik-Schnirelmann method for invariant functionals and relative cohomological index theories. In Méthodes topologiques en analyse non linéaire. A. Granas (ed.) Sémin. Math. Sup. No. 95. Montréal, 41-70.

[19] Golubitsky, M. and Stewart, I. With an appendix by J. E. Marsden. [1987] Generic bifurcation of Hamiltonian systems with symmetry. Physica D, 24, 391-405.

[20] Golubitsky, M., Stewart, I., and Schaeffer, D.G. [1988] Singularities and Groups in Bifurcation Theory: Vol. II. Applied Mathematical Sciences, Vol. 69, Springer-Verlag.

[21] Guillemin, V., Lerman, E., and Sternberg, S. [1996] Symplectic Fibrations and Multiplicity Diagrams. Cambridge University Press.

[22] Guillemin, V. and Pollack, A. [1974] Differential Topology. Prentice-Hall.

[23] Guillemin, V. and Sternberg, S. [1984] A normal form for the moment map. In Differential Geometric Methods in Mathematical Physics. S. Sternberg ed. Mathematical Physics Studies, 6. D. Reidel Publishing Company.

[24] Guillemin, V. and Sternberg, S. [1984] Symplectic Techniques in Physics. Cambridge University Press.

[25] Hofer, H., and Zehnder, E. [1995] Symplectic invariants and Hamiltonian dynamics. In The Floer Memorial Volume. Pages 525-544. Taubes, C. H., Weinstein, A., and Zehnder, E. (editors). Birkhäuser Verlag, Progress in Mathematics Series, number 133.

[26] Horn, J. [1903] Beiträge zur Theorie der kleinen Schwingungen. Z. Math. Phys., 48, 400-434.

[27] Humphreys, J. E. [1972] Introduction to Lie Algebras and Representation Theory. Graduate Texts in Mathematics, no. 9. Springer-Verlag.

[28] Kawakubo, K. [1991] The Theory of Transformation Groups. Oxford University Press.

[29] Kirwan, F. [1984] Cohomology of quotients in symplectic and algebraic geometry. Mathematical Notes, 31. Princeton University Press. Princeton, 1984.

[30] Kirwan, F. [1988] The topology of reduced phase spaces of the motion of vortices on a sphere. Physica D, 30, 99-123. 
[31] Krein, M. G. [1950] A generalization of several investigations of A. M. Liapunov on linear differential equations with periodic coefficients. Dokl. Akad. Nauk. SSSR, 73, 445-448.

[32] Laurent-Polz, F.[2002] Point vortices on the sphere: a case with opposite vorticities. Nonlinearity, 15, $143-171$.

[33] Leonard, N. E.[1997] Stability of a bottom-heavy underwater vehicle. Automatica, 33(3), 331-346.

[34] Leonard, N. E. and Marsden, J. E.[1997] Stability and drift of underwater vehicle dynamics: mechanical systems with rigid motion symmetry. Physica D, 105, 130-162.

[35] Lerman, E. and Singer, S.F. [1998] Stability and persistence of relative equilibria at singular values of the moment map. Nonlinearity, 11, 1637-1649.

[36] Lerman, E. and Tokieda, T. F. [1999] On relative normal modes. C. R. Acad. Sci. Paris Sér. I Math., 328, 413-418.

[37] Lewis, D., Ratiu, T. S., Simo, J.C. and Marsden, J.E. [1992] The heavy top: a geometric treatment. Nonlinearity, 5, 1-48.

[38] Liapunov, M. A. [1947] Problème Général de la Stabilité du Mouvement. Annals of Mathematical Studies, number 17. Princeton University Press. Translation from the original in Russian appeared in the Annales de la Faculté des Sciences de Toulouse, Second Series, Volume 9 (1907).

[39] Lim, C., Montaldi, J. A., and Roberts, R.M. [2001] Relative equilibria of point vortices on the sphere. Physica D, 148, 97-135.

[40] Marsden, J.E. and Scheurle, J. [1993a] Lagrangian reduction and the double spherical pendulum. Z. Angew. Math. Phys., 44, 17-43.

[41] Marle, C.-M. [1985] Modéle d'action hamiltonienne d'un groupe the Lie sur une variété symplectique. Rend. Sem. Mat. Univers. Politecn. Torino, 43(2), 227-251.

[42] Marsden, J.E. and Scheurle, J. [1993a] Lagrangian reduction and the double spherical pendulum. Z. Angew. Math. Phys., 44, 17-43.

[43] Marsden, J.E., and Weinstein, A. [1974] Reduction of symplectic manifolds with symmetry. Rep. Math. Phys., 5(1), 121-130.

[44] Marzantowicz, W. [1989] A G-Lusternik-Schnirelamn category of space with an action of a compact Lie group. Topology, 28, 403-412.

[45] McDuff, D. and Salamon, D. [1998] Introduction to Symplectic Topology. Oxford Mathematical Monographs, Clarendon Press, Oxford. Second Edition.

[46] Montaldi, J. [1997] Persistence and stability of relative equilibria. Nonlinearity, 10, 449-466.

[47] Montaldi, J. [1997] Persistance d'orbites périodiques relatives dans les systèmes hamiltoniens symétriques. C. R. Acad. Sci. Paris Sér. I Math., 324, 553-558.

[48] Montaldi, J.A. and Roberts, R.M. [1999] Relative equilibria of molecules. J. Nonlin. Sci., 9, 53-88.

[49] Montaldi, J. A., Roberts, R.M., and Stewart, I.N. [1988] Periodic solutions near equilibria of symmetric Hamiltonian systems. Phil. Trans. R. Soc. Lond. A, 325, 237-293.

[50] Moser, J. [1958] New aspects in the theory of stability of Hamiltonian systems. Comm. Pure Appl. Math., 11, 81-114.

[51] Moser, J. [1976] Periodic orbits near an equilibrium and a theorem by Alan Weinstein. Comm. Pure Appl. Math., 29, 727-747.

[52] Ortega, J.-P. [1998] Symmetry, Reduction, and Stability in Hamiltonian Systems. Ph.D. Thesis. University of California, Santa Cruz. June, 1998.

[53] Ortega, J.-P. and Ratiu, T.S. [1998] Singular reduction of Poisson manifolds. Letters in Mathematical Physics, 46, 359-372.

[54] Ortega, J.-P. and Ratiu, T.S. [1999] Stability of Hamiltonian relative equilibria. Nonlinearity, 12(3), 693720 .

[55] Ortega, J.-P. and Ratiu, T. S. [2003] Momentum Maps and Hamiltonian Reduction. To appear in Birkhäuser, Progress in Mathematics. 
Juan-Pablo Ortega: Relative normal modes for nonlinear Hamiltonian systems

[56] Ortega, J.-P. and Ratiu, T. S. [2000] The dynamics around stable and unstable Hamiltonian relative equilibria. Preprint.

[57] Palais, R. [1961] On the existence of slices for actions of non-compact Lie groups. Ann. Math., 73, $295-323$.

[58] Patrick, G.W. [1992] Relative equilibria in Hamiltonian systems: the dynamic interpretation of nonlinear stability on a reduced phase space. J. Geom. Phys., 9, 111-119.

[59] Patrick, G.W., Roberts, M., and Wulff, C. [2002] Stability of Poisson equilibria and Hamiltonian relative equilibria by energy methods. Preprint http://arXiv.org/abs/math.DS/0201239

[60] Roberts, M., Wulff, C., and Lamb, J. S. W. [2002] Hamiltonian systems near relative equilibria. J. Differential Equations, 179(2), 562-604.

[61] Schwartz, J. T. [1969] Nonlinear Functional Analysis. Gordon and Breach.

[62] Sjamaar, R. and Lerman, E. [1991] Stratified symplectic spaces and reduction. Ann. of Math., 134, 375-422.

[63] Smale, S. [1970] Topology and mechanics. Inv. Math. 10, 305-331; 11, 45-64.

[64] van der Meer, J. C. [1985] The Hamiltonian Hopf Bifurcation. Lecture Notes in Mathematics, 1160. Springer Verlag.

[65] van der Meer, J. C. [1990] Hamiltonian Hopf bifurcation with symmetry. Nonlinearity, 3, 1041-1056.

[66] Vanderbauwhede, A. and van der Meer, J. C. [1995] General reduction method for periodic solutions near equilibria. In Normal Forms and Homoclinic Chaos, pages 273-294. Langford, W. F. and Nagata, W. eds. Fields Institute Communications, 4.

[67] Weinstein, A. [1973] Normal modes for nonlinear Hamiltonian systems. Inventiones Math., 20, 47-57.

[68] Weinstein, A. [1977] Symplectic $V$-manifolds, periodic orbits of Hamiltonian systems, and the volume of certain Riemannian manifolds. Comm. Pure Appl. Math., 30, 265-271.

[69] Williamson, J. [1936] On the algebraic problem concerning the normal forms of linear dynamical systems. Amer. J. Math., 58, 141-163. 